\title{
Online Resource Provisioning for Wireless Data Collection
}

\author{
YU LIU, JOSHUA COMDEN, ZHENHUA LIU, and YUANYUAN YANG, \\ Stony Brook University, United States
}

\begin{abstract}
Wireless data collection requires a sequence of resource provisioning decisions due to the limited battery capacity of wireless sensors. The corresponding online resource provisioning problem is challenging. Recently, many prediction methods have been proposed that can be used to benefit the performance of various systems through their incorporation. Therefore, in this article, we focus on online resource provisioning problems with short-term predictions motivated by the wireless data collection problem. Specifically, we design separate online algorithms for systems in which the state evolves in either a stationary manner or an arbitrarily determined manner and prove their performance bounds where their bounds improve as the amount of available predictions increases. Additionally, we design a meta-algorithm that can choose which online algorithm to implement at each point in time, depending on the recent behavior of the system environment. The practical performances of the proposed algorithms are corroborated in trace-driven numerical simulations of data collection of shared bikes. Additionally, we show that the performance of our meta-algorithm in various system environments can be better than that of the single best algorithm chosen in hindsight.
\end{abstract}

CCS Concepts: • Theory of computation $\rightarrow$ Online algorithms; Design and analysis of algorithms; • Applied Computing $\rightarrow$ Decision analysis;

Additional Key Words and Phrases: Online resource provisioning, short-term predictions, resource constraints, competitive analysis

ACM Reference format:

Yu Liu, Joshua Comden, Zhenhua Liu, and Yuanyuan Yang. 2021. Online Resource Provisioning for Wireless Data Collection. ACM Trans. Sen. Netw. 18, 1, Article 7 (September 2021), 27 pages.

https://doi.org/10.1145/3470648

\section{INTRODUCTION}

Real-world applications in many areas including wireless sensor systems require a sequence of resource provisioning decisions in time to be made with the goal of maximizing the overall revenue. Depending on the time frame and application, revenue function is time dependent and it is often unrealistic to assume that all future revenue functions can be known beforehand. Thus, decisions need to be made step by step without full future information while still making a satisfactory level of achievement toward the goal, even if it is the not perfect sequence of resource provisioning decisions made in hindsight.

This research was partially funded by NSF grants CNS-1617698, CNS-1717588, CNS-1730128, and CNS-1919752.

Authors' address: Y. Liu, J. Comden, Z. Liu, and Y. Yang, Stony Brook University, 100 Nicolls Rd, Stony Brook, NY 11794; emails: yu.liu.3@stonybrook.edu, joshuacomden@gmail.com, zhenhua.liu@stonybrook.edu, yuanyuan.yang@ stonybrook.edu.

Permission to make digital or hard copies of all or part of this work for personal or classroom use is granted without fee provided that copies are not made or distributed for profit or commercial advantage and that copies bear this notice and the full citation on the first page. Copyrights for components of this work owned by others than ACM must be honored. Abstracting with credit is permitted. To copy otherwise, or republish, to post on servers or to redistribute to lists, requires prior specific permission and/or a fee. Request permissions from permissions@acm.org.

(C) 2021 Association for Computing Machinery.

1550-4859/2021/09-ART7 \$15.00

https://doi.org/10.1145/3470648 
A specific application of online resource provisioning is as follows. A wireless sensor located in a shared bike equipped with a battery transmits data to base stations under time-varying signal attenuation rates, and it needs to decide the amount of energy to use at each time slot with the goal of maximizing the size of collected data [30]. The system state of each time slot is the current signal attenuation rate between the shared bike and the nearest base station, and the revenue of each time slot is the current data transfer rate, which is a function of the current system state and the resource provisioning decision. The data collected by sharing data are valuable for the operator to optimize its service, e.g., optimization of parking location placement [38], re-balancing bike sharing systems [24], and collection of malfunctioning bikes [14].

The research area that develops algorithms that make these sequential decisions to achieve their desired goals as best as possible is called Online Optimization [1, 15, 23]. In this article, we focus on online resource provisioning. The decision of online resource provisioning problems involves choosing how much of a specific resource to use at each point in time. Naturally, there is a constraint on resources, which puts a limit on how much of the resource can be used in total during the system's operating time. If a decision uses more of the resource at one point, then a decision at some other point in time in the future will need to use less if the resource constraint is to be respected. Resource constraints can be found in many online resource provisioning applications, such as optimally allocating online advertisement space to budget-constrained advertisers [27]; battery allocation for wireless sensor networks under cost constraints [26]; dynamic resource management for high-speed railway wireless communications with time average power constraint [34]; scheduling for incentive WiFi offloading with energy constraint [17]; and quality-oriented rate control and resource allocation in dynamic OFDMA networks with base station transmit power constraint [18].

Recently, a formulation of inventory constrained online optimization has been studied and a state-of-the-art result has been established by Lin et al. [23]. Specifically, they developed an algorithm named CR-Pursuit and showed that it achieves a competitive ratio of $c(\ln \theta+1)$, where $c$ and $\theta$ are constants that depend on properties of the objective functions. Despite these strong theoretical guarantees, CR-Pursuit may still perform poorly in some realistic scenarios. See Section 2 for a practical demonstration.

Typically in the past, online resource provisioning took the very pessimistic view that nothing can be known about the future. However, recently, one promising direction that has been gaining traction is to include predictions as part of the input for online algorithms, as shown in $[8,19]$. For instance, short-term bandwidth predictions can help improve the quality of video streaming for cellular networks [39]. Even noisy predictions can be combined with various control algorithms dynamically for cloud resource provisioning [8].

However, even with their success in other online optimization problems, predictions have not yet been explored for online resource provisioning. This could be due to the fact that it is not entirely clear how predictions can and should be used, and how much they can help. For example, when predictions are given for a limited look-ahead window in a system that is relatively stationary, they may reveal information about the system beyond the look-ahead window because statistics from the predictions can be used to make inferences for the distant future. On the other hand, if the system changes in an arbitrary or adversarial fashion, the predictions may reveal no new information about the system beyond the look-ahead window. Depending on the environment that the online algorithm is operating in, the predictions may be used in different ways. Therefore, the goal of this article is to quantitatively understand the benefits of predictions in online resource provisioning with a resource constraint. Specifically, our aim is to develop online algorithms that use predictions and respect the resource constraint in different system environments. 


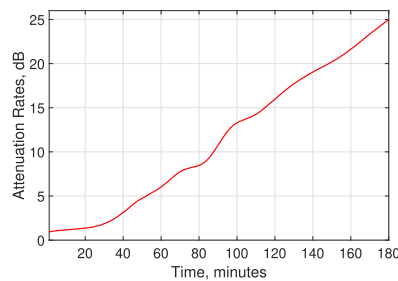

(a) Trace 1

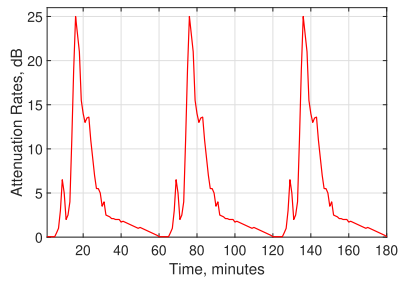

(b) Trace 2

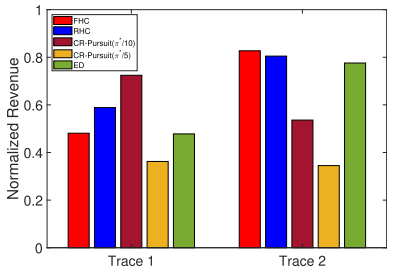

(c) Algorithm Performances

Fig. 1. Attenuation rate vs. time for (a) an increasing trace and (b) a spiked trace. The spiked trace was generated by repeating the trace from [28] three times. The increasing trace was also made from [28], but using only the second half by scaling up the attenuation rates and the timescale, and then reversing it in time. (c) is the revenue for different online algorithms applied to the traces, normalized by the offline optimal solution: FHC (red), RHC (blue), CR-Pursuit( $\left.\pi^{*} / 10\right)$ (brown), CR-Pursuit $\left(\pi^{*} / 5\right)$ (tan), and ED (green).

We summarize our main contributions as follows:

(1) We propose a framework for incorporating predictions into the online resource provisioning with resource constraints and short-term predictions under different system environments: stationary and arbitrarily determined (Section 3).

(2) We design two online algorithms that incorporate predictions into their decisions and prove their performance guarantees, each designed for a different specific system environment (Section 4).

(3) We design a simple meta-algorithm that can combine the decisions of many individual online algorithms that can adapt to any environment it is used in (Section 5).

(4) We evaluate the performance of proposed algorithms with trace-based simulations of data collection of shared bikes and show that our algorithms outperform the existing algorithms (Section 6).

In the next section, we demonstrate a real-world example of how existing algorithms are not well suited to handle different system environments.

\section{MOTIVATIONAL EXAMPLE}

The objective of this simple example is to show how existing online algorithms that are either designed for resource constraints or including predictions, but not both, have mixed results in different system environments.

We use the problem situation of data collection of shared bikes where the sensors in shared bikes need to send data to base stations. The goal is to send as much data as possible within a specific operating time by deciding how much energy the sensor is using in each time slot. However, the attenuation rate between a sensor and the nearest base station changes in time and determines how much data can be transferred, depending on the amount of energy the sensor is using. The lower the attenuation rate, the more data that can be transferred with the same energy. However, the sensor's total energy usage is limited by the battery supplying the energy. More details on this setup can be found in Section 6. We study two attenuation rate traces: a gradually increasing scenario (Figure 1(a)) and a scenario with repeating spikes (Figure 1(b)).

The performance in terms of data transferred (labeled as revenue) for different online optimization algorithms is shown in Figure 1(c). We use a naive benchmark called Equally Distributed $(E D)$, which simply equally divides the energy stored in the battery over the operating time. As can be expected, $E D$ performs better in the scenario with the repeating pattern. 
Table 1. Important Notations

\begin{tabular}{c|c}
\hline$T$ & Number of time slots \\
\hline$z_{t}$ & System state at time slot $t$ \\
\hline$Z$ & Set of system states, i.e., $\{z(1), z(2), \ldots, z(I)\}$ \\
\hline $\mathbf{Z}$ & Set of system state sequences, i.e., $Z^{T}$ \\
\hline $\mathbf{Z}$ & System state sequence, $\mathbf{z} \in \mathbf{Z}$ \\
\hline$x_{t}$ & Resource provisioning decision at time slot $t$ \\
\hline$R(\cdot, \cdot)$ & Revenue function \\
\hline$r_{t}$ & Revenue at time slot $t$, i.e., $R\left(x_{t}, z_{t}\right)$ \\
\hline$p(t)$ & $\left.\frac{\partial R\left(x_{t}, z_{t}\right)}{\partial x_{t}}\right|_{x_{t}=0}$, base price at time slot $t$ \\
\hline$m, M$ & Lower and upper bounds of $p(t), t \in[T]$ \\
\hline$\theta$ & The ratio of $M / m$ \\
\hline$\eta^{*}$ & Optimal offline revenue of the system \\
\hline
\end{tabular}

CR-Pursuit [23], an algorithm with theoretical guarantees for online optimization problems with inventory constraints but that does not use predictions, was run at two different settings $\left\{\frac{\pi^{*}}{10}, \frac{\pi^{*}}{5}\right\}$, where $\pi^{*}$ is the smallest competitive ratio proved by [23]. In both of the traces, either one or both of the CR-Pursuit settings performed worse than the naive benchmark ED. This shows that even with its proven guarantees, there are system environments where it can perform poorly in practice relative to other algorithms.

We also tested two online algorithms, Receding Horizon Control (RHC) and Fixed Horizon Control (FHC) [22], that use predictions but are not designed for online optimization problems with inventory constraints. We modified them to the problem by giving them a miniaturized version of the inventory constraint that is scaled to the size of the look-ahead window. We see that in Trace 2 they both performed better than either of the CR-Pursuit settings. But in Trace 1 they both performed worse than one of the settings of CR-Pursuit and better than the other. This shows both their performance potential in being applied to this problem and their possible shortcomings.

Thus, the goal in this article is to combine the practical performance benefits of using predictions with the theoretical guarantees of CR-Pursuit to make new online algorithms that contain both, depending on the system environment.

\section{PROBLEM FORMULATION}

In this section, we formulate the general mathematical model for the online resource provisioning problem with resource constraints and short-term predictions. A real-world application of the problem can be found in Section 6. Some important notations are summarized in Table 1.

\subsection{System Model}

Consider a system that operates within a finite time horizon $T$ and a controller that makes a resource provisioning decision for each time slot $t \in\{1,2, \ldots, T\}$. At the beginning of every time slot $t$, the controller receives predictions of the system states within a given prediction window of size $w$ and uses those predictions to make a decision for acquiring revenue. The decision being made is the amount of the system's finite resource consumed during the current time slot. The goal is to maximize the total system revenue subject to the total resource available from the start. We assume that the time horizon $T$ is known to the system controller beforehand.

System States. At each time slot $t$, the system is in state $z_{t}$, which determines the corresponding revenue function. In the system of data collecting of shared bikes that we simulate in Section 6 , 
the system state of time slot $t$ is the signal attenuation of a wireless channel between a sensor of a shared bike and its nearest base station, and the signal attenuation can determine the data rate, the revenue of the system. The revenue of the same amount of resources is different under different system states; e.g., the data rate of a wireless channel with the same signal power is different under different attenuation rates [9]. The system states can also be prices of a resource over time, signal attenuation rates, noise power of a wireless telecommunication channel, and so forth, depending on the application. The state space can be continuous or discrete. In this article, we focus on the discrete case, but our analytical results can be easily adapted to the continuous case. Each state $z_{t}$ is drawn from a countable set $Z$ with $I$ different states, denoted by $\{z(1), z(2), \ldots, z(I)\}$. A specific sequence of states for the whole time horizon $T$ is denoted by $z$, with all possible such sequences being contained within the set $\mathrm{Z} \triangleq Z^{T}$, i.e., $\mathbf{z} \in \mathrm{Z}$.

Resource Provisioning Decisions. At the beginning of each time slot $t$, the system controller makes a resource provisioning decision $x_{t}$, which is the amount of resource used at the current time slot. We consider a constrained problem where $x_{t}$ is chosen from a compact decision space $\mathcal{A}_{z_{t}}=$ $\left[0, \delta_{z_{t}}\right]$ with the upper bound $\delta_{z_{t}}$ being possibly associated with the system's state $z_{t}$. For example, if $x_{t}$ represents energy usage sourced from a battery, $\delta_{z_{t}}$ could be the fastest rate of discharge possible from the battery.

Resource Constraint. When the system operates, there exists a finite total starting resource budget $\Delta$ from which all the decisions are sourced. For example, this could be the total amount of energy stored in a battery of a wireless device. Once the resource is completely depleted by the decisions, no more nonzero decisions can be made afterward. The size of the resource constrains the feasible sequence of decisions by limiting its total accumulated decision values over the time horizon $T$, which is stated specifically by $\sum_{t=1}^{T} x_{t} \leq \Delta$, where $x_{t} \in \mathcal{A}_{z_{t}}$ for all $t \in\{1,2, \ldots, T\}$. The total resource $\Delta$ is known to the controller in advance.

Revenue. At each time slot $t$, the system controller receives the revenue of $r_{t}=R\left(x_{t}, z_{t}\right)$ after performing the decision $x_{t}$ in system state $z_{t}$, where $R(\cdot, \cdot)$ is the revenue function $R: \mathcal{A}_{z_{t}} \times$ $\{z(1), z(2), \ldots, z(I)\} \rightarrow \mathbb{R}$. The total revenue over the time horizon is the objective value that the system wants to maximize. The revenue in the system we simulate in Section 6 is the total amount of data collected by a sensor in a shared bike over the operating time horizon. The revenue $r_{t}$ can also be the size of energy charged to an electric vehicle [35], the quality of service of a network service [32], and so forth, depending on the application. Similarly to the literature [23, 29], we make some weak assumptions. We assume that the revenue functions $R\left(x_{t}, z_{t}\right)$ satisfy the following conditions:

- $R\left(x_{t}, z_{t}\right)$ is concave, increasing, and differentiable with respect to $x_{t} \in \mathcal{A}_{z_{t}}$, for all $z_{t}$.

- $R\left(0, z_{t}\right)=0$ for $z_{t} \in Z$.

- $\left.p(t) \triangleq \frac{\partial R\left(x_{t}, z_{t}\right)}{\partial x_{t}}\right|_{x_{t}=0} \in[m, M]$.

The first condition implies that for a given system state, the more resource that is consumed results in more revenue that is gained with diminishing marginal returns. The concave assumption comes from the general law of diminishing marginal utility and is a typical assumption made in the literature (see $[23,29])$. The second condition says that there cannot be any revenue without using some resource. $\left.p(t) \triangleq \frac{\partial R\left(x_{t}, z_{t}\right)}{\partial x_{t}}\right|_{x_{t}=0}$ is the base price, which is the marginal revenue when zero resource is being used. The third property bounds base prices, marginal revenues at the origin, to be within the interval $[m, M]$. Let the ratio of the largest possible base price to the smallest be denoted by $\theta \triangleq M / m$, which we assume is known by the system controller in advance as was also done by [23]. 


\subsection{Online Optimization Problem}

With the goal of maximizing the total system revenue while simultaneously respecting the given starting resource $\Delta$, we have the following optimization problem:

$$
\begin{array}{cl}
\max _{x_{t}, t \in[T]} & \sum_{t=1}^{T} R\left(x_{t}, z_{t}\right) \\
\text { s.t. } & x_{t} \in \mathcal{A}_{z_{t}} \text { for } t \in\{1,2, \ldots, T\} \\
& \sum_{t=1}^{T} x_{t} \leq \Delta .
\end{array}
$$

Let $\eta^{*}$ be the optimal offline objective value of $\mathrm{P} 1$.

However, it is unrealistic to assume that the system states over the entire operating time are known beforehand for a controller to simply implement the optimal offline solution. Instead, the controller will have to make its decisions time slot by time slot as the system states evolve in an online manner without full knowledge of the states in the future. Thus, we do allow some limited knowledge of the future through short-term predictions.

In time slot $t$, the system controller receives predictions of the next $w$ states $z_{t}, z_{t+1}, \ldots, z_{t+w-1}$ and then chooses a resource provisioning decision $x_{t}$ based on an online algorithm. After the resource provisioning decision is implemented, the system's state $z_{t}$ is revealed. Our goal is to design an online algorithm for the system controller that maximizes the overall revenue gained and respects the resource constraint by utilizing the predictions.

\subsection{System Environments}

Before making a decision, the system controller receives predictions for the next $w$ system states $z_{t}, z_{t+1}, \ldots, z_{t+w-1}$, where $w \leq T$ is the prediction window size. For the case near the end of the time horizon when less than $w$ time slots remain, we simply assume that the predictions of the states beyond $T$ start again from the beginning, i.e., $z_{t}=z_{t \% T+1}$ if $t>T$, where $\%$ is the modulo operator. How the predictions get used by the controller depends on the system environment that it is being operated in. We explore two distinct system environments that behave in fundamentally opposite ways.

Stationary Environment. In some real-world systems, the evolution of system states over time can be modeled as an i.i.d. process of randomly selecting an element from the set $Z$, e.g., the connectivity of links in some wireless networks can be viewed as an i.i.d process over time [20,25]. If $z_{t}$ is i.i.d. over time, we denote $p_{i}$ as the time-invariant probability that $z_{t}=z(i)$ and $\mathbf{p}=\left(p_{1}, p_{2}, \ldots, p_{I}\right)$ as the system state probability distribution. The predictions in this environment give more information about the system beyond the prediction window since the relative frequencies of states in the prediction window can also be considered as an estimation of system state probability distribution $\mathbf{p}$. An example system with an arbitrarily determined environment is to maximize the time cumulative availability of virtual network functions (VNFs) under cost constraints [32], where the system states, failure probabilities of VNFs, are assumed to be arbitrarily determined.

Arbitrarily Determined Environment. However, in other systems, such as price of energy [35] and data center workloads [33], the evolution of states is often non-stationary and does not follow an i.i.d. process. In this environment, we assume that the states are not generated according to any rule so that the predictions only give information within the prediction window and cannot be used to infer anything else about the system. In fact, the states could be adversarially determined against the controller's decisions in the worst case. One example system with arbitrarily 


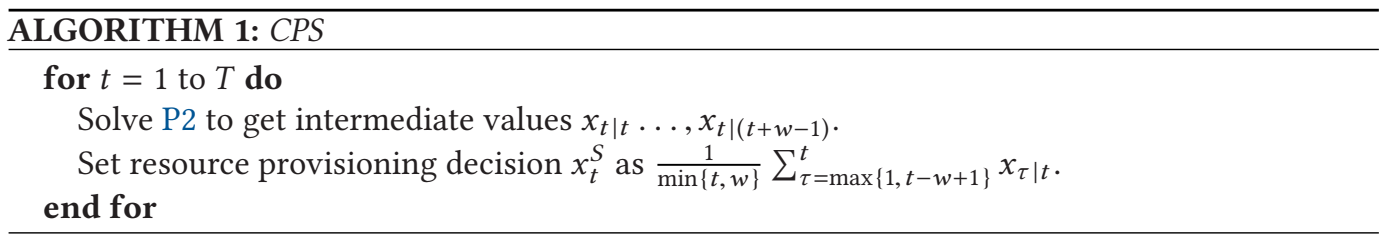

determined environment is to maximize the time cumulative availability of VNFs under cost constraints [32], where the system states, failure probabilities of VNFs, are assumed to be arbitrarily determined.

These two environments can be thought of as extreme cases as to how much information about the system can be inferred from its past states and predictions. Most real-world systems are located in between these two extremes and possibly vary in time whether it is closer to a stationary or an arbitrarily determined environment.

There have been lots of algorithms focusing on predicting. As in the previous work [7], we focus on designing online algorithms assuming predictions are available. In Section 6, we conduct simulations under predictions with different predicting errors.

\section{ONLINE ALGORITHMS}

In this section, we design two separate online algorithms and prove their performance, each for a different environment of system state evolution: stationary and arbitrarily determined.

\subsection{Systems with Stationary States}

Here we introduce a novel algorithm, called Control Policy for a system with Stationary system states (CPS), for systems with i.i.d. system states, and provide performance analysis for CPS. The main idea of $C P S$ is to make system decisions based on system states in the prediction window only. Since the system is stationary, the distribution of system states within a prediction window, to a certain extent, can represent the overall distribution of system states. CPS combines the idea of Averaging Fix Horizon Control (AFHC) [22] to incorporate predictions, where AFHC is an online algorithm for online optimization problems without budget constraints choosing online decisions by combining the decisions made under different prediction windows. When the system is stationary, $\mathrm{AFHC}$ can combine outputs from multiple prediction windows to make decisions. By doing so, it utilizes more information of the system states and smoothes the decisions. As for the resource constraint, the budget gets amortized evenly across the time horizon.

CPS is formally stated in Algorithm 1. At the beginning of every time slot $t$, it observes $z_{t}, z_{t+1}$, $\ldots, z_{t+w-1}$ and solves the following convex optimization problem, P2:

$$
\begin{array}{cl}
\max _{x_{t \mid t}, x_{t \mid(t+1)}, \ldots, x_{t \mid(t+w-1)}} & \sum_{\tau=t}^{t+w-1} R\left(x_{t \mid \tau}, z_{\tau}\right) \\
\text { s.t. } & x_{t \mid \tau} \in \mathcal{A}_{z_{\tau}} \text { for } \tau \in\{t, \ldots, t+w-1\} \\
& \sum_{\tau=t}^{t+w-1} x_{t \mid \tau} \leq \frac{w}{T} \Delta .
\end{array}
$$

With an abuse of notation, we use $x_{t \mid t}, x_{t \mid t+1}, \ldots, x_{t \mid(t+w-1)}$ to denote the optimal solution of P2. The optimization problem $\mathrm{P} 2$ is designed to optimally solve for the miniaturized version of $\mathrm{P} 1$ that only extends out $w$ instead of $T$ time slots and with a resource $\frac{w}{T} \Delta$ that is reduced proportionally. 
After solving P2, CPS makes the decision as $x_{t}^{S}$, which is the average decision associated with time slot $t$ from the current and the previous $w-1$ calculations of $\mathrm{P} 2$ :

$$
x_{t}^{S}=\frac{1}{\min \{t, w\}} \sum_{\tau=\max \{1, t-w+1\}}^{t} x_{\tau \mid t} .
$$

Because each solution of $\mathrm{P} 2$ at different time slots sees a slightly different set of states, averaging them together for a particular time slot's resource smoothes out the differences between them to give a more balanced decision. This is advantageous in a stationary environment since the states $z(t)$ are chosen i.i.d. over time, which means that $\mathbb{E}\left[x_{t \mid \tau}\right]$ and $\mathbb{E}\left[R\left(x_{t \mid \tau}, z_{\tau}\right)\right]$ remain constant across all $t$ and $\tau \in\{t, t+1, \ldots, t+w-1\}$.

Next we analyze the competitive performance of CPS and use $\eta^{S}$ to denote the total amount of revenue achieved by CPS. First we show its average-case performance by proving a lower bound on the expected revenue of CPS compared to the expected offline optimal revenue and an upper bound on its expected resource consumption. Note that $\eta^{*}$ is the optimal offline revenue.

Theorem 4.1. For P1 with state distribution $\mathbf{p}=\left(p_{1}, p_{2}, \ldots, p_{I}\right)$, using CPS, we have that

(1) $\mathbb{E}\left[\eta^{S}\right] \geq \mathbb{E}\left[\eta^{*}\right] \cdot \frac{1-\epsilon}{1+\epsilon} \cdot\left(1-\sum_{i=1}^{I} 2 e^{-\frac{w \epsilon^{2} p_{i}}{3}}\right)$ for all $\epsilon \in(0,1)$.

(2) $\sum_{t=1}^{T} E\left[x_{t}^{S}\right] \leq \Delta$.

The proof is found in Appendix A. The proof idea consists of three parts. First, for system states within the same prediction window, Lemma 1 in Appendix A.1 shows that given the window size $w$ and $\epsilon \in(0,1)$, the probability of $\exists i$ such that $\left|\sum_{\tau=t}^{t+w-1} \mathbb{1}_{i}\left(z_{\tau}\right) / w-p_{i}\right| \geq p_{i} \epsilon$ is not greater than $\sum_{i=1}^{I} 2 e^{-w \epsilon^{2} p_{i} / 3}$. Then, Lemma 2 in Appendix A.2 shows that for system states in the same prediction window satisfying $\left|\sum_{\tau=t}^{t+w-1} \mathbb{1}_{i}\left(z_{\tau}\right) / w-p_{i}\right|<p_{i} \epsilon$, the system resources made by $\mathrm{P} 2$ have a time average revenue no less than $\frac{1}{T} E\left[\eta^{*}\right] \frac{1-\epsilon}{1+\epsilon}$. Finally, in Appendix A.3, we combine Lemma 1 and Lemma 2 to get Theorem 4.1.

As we can see from Theorem 4.1, the lower bound of $\mathbb{E}\left[\eta^{S}\right]$ increases as the prediction window size $w$ increases. For any $\epsilon \in(0,1), \mathbb{E}\left[\eta^{S}\right]$ converges to $\frac{1-\epsilon}{1+\epsilon} \cdot \mathbb{E}\left[\eta^{*}\right]$ as $w \rightarrow \infty$. When $\theta$, the ratio between the maximum and minimum base price, is large, the existing CR-Pursuit algorithm [23] has poor performance, whereas the lower bound of revenue in Theorem 4.1 does not depend on $\theta$.

In addition to comparing the expected revenue of CPS to the expected offline optimal revenue, we also give its lower bound with high probability compared to the offline optimal revenue itself in the following proposition.

Proposition 1. Under CPS, for all $\epsilon \in(0,1)$ and all $\psi \in(0,1), \mathbb{E}\left[\eta^{S}\right] \geq \eta^{*} \cdot \frac{1-\epsilon}{1+\epsilon} \cdot \frac{1-\psi}{1+\psi} \cdot(1-$ $\left.\sum_{i=1}^{I} 2 e^{-\frac{w \epsilon^{2} p_{i}}{3}}\right)$ with probability no less than $\left(1-\sum_{i=1}^{I} 2 e^{-\frac{T \psi^{2} p_{i}}{3}}\right)$.

The proof is found in Appendix A.4. The proof idea of this proposition consists of two steps. We first show that the probability of $E\left[\eta^{*}\right]<\eta^{*} \frac{1-\psi}{1+\psi}$ is less than $\sum_{i=1}^{I} 2 e^{-\frac{T \psi^{2} p_{i}}{3}}$. Then, we combine this result with that from Theorem 4.1. From Proposition 1, we have a similar conclusion as Theorem 4.1 in that $\mathbb{E}\left[\eta^{S}\right]$ converges to $\frac{1-\epsilon}{1+\psi} \cdot \eta^{*}$ as $w \rightarrow \infty$. Additionally, it shows us that the probability of $\mathbb{E}\left[\eta^{S}\right]$ satisfying this lower bound approaches 1 as the time horizon $T$ approaches infinity.

While Theorem 4.1 shows us that the expected resource consumption of CPS satisfies the resource constraint, we are also concerned on what the worst-case resource consumption could be. The following proposition gives this upper bound. 
Proposition 2. Under CPS, the total amount of resource consumed satisfies that $\sum_{t=1}^{T} x_{t}^{S} \leq$ $(1+\log (w-1)) \frac{\Delta}{T / w}+\Delta$.

The proof is found in Appendix A.5. This proposition shows us that as the time horizon $T$ increases, its worst-case resource consumption approaches the initially given resource of $\Delta$.

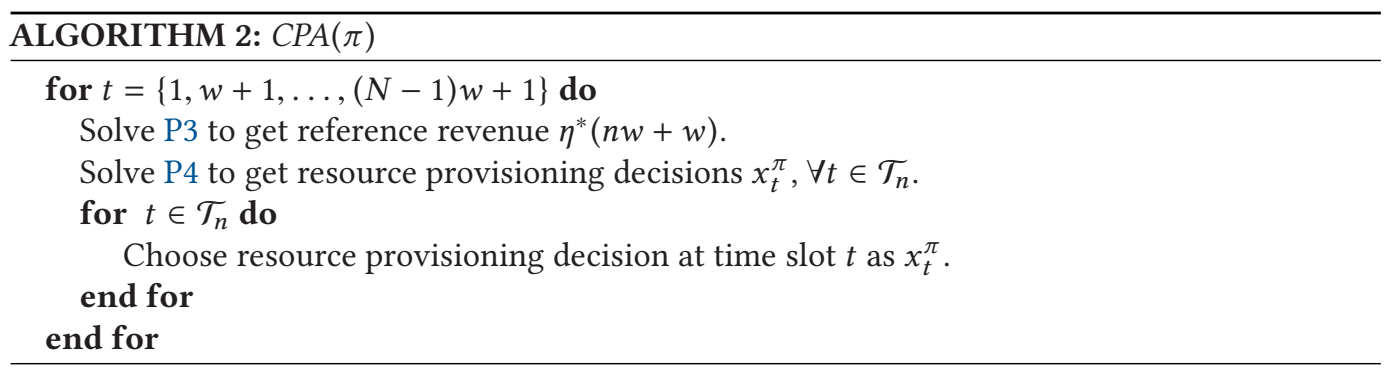

\subsection{Systems with Arbitrarily Determined States}

For systems with states that are arbitrarily determined, we introduce an algorithm called Control Policy for a system with Arbitrary system states (CPA) and prove its competitive ratio. Unlike in the environment for $C P S, C P A$ cannot use its predictions to make an inference about the distribution of states beyond the prediction window. The main idea of $C P A$ comes from combining the CR-Pursuit algorithm [23] with the FHC algorithm [7] into one that can both manage its resource consumption and utilize predictions of future states. CR-Pursuit is an online algorithm for online optimization problems with budget constraints and without predictions, and FHC is an online algorithm for online optimization problems with budget constraints, in which choosing online decision computes decision under a given prediction window and implements all actions in the prediction window. When the system is non-stationary, mixing decisions in AFHC may result in slow response to sudden system changes, and hence poor performance guarantee. Combining CR-Pursuit and the idea of FHC to incorporate predictions can achieve an algorithm with a theoretical performance guarantee.

$C P A$ has a single parameter $\pi>1$ that adjusts how aggressively it tries to match the optimal reward for a modified version of $\mathrm{P} 1$ that had its time horizon cut short (see Problem P3). $C P A(\pi)$ represents $C P A$ with parameter $\pi$. For simplicity, we assume that time horizon $T$ is a multiple of the prediction window $w$, i.e., $T=N \cdot w$, where $N$ is an integer, and use $\mathcal{T}_{n}$ to represent the set of time slots $\{n \cdot w+1, n \cdot w+2, \ldots,(n+1) \cdot w\}$ for the $n$th round of predictions, where $n \in\{0,1, \ldots, N-1\}$.

$C P A$ is formally stated in Algorithm 2. Let $\eta^{*}(0)=0$ and let $\eta^{*}(t), t \geq 1$ be the optimal objective value of the following convex optimization problem:

$$
\begin{aligned}
\max _{x_{\tau}, \tau \in[t]} & \sum_{\tau=1}^{t} R\left(x_{\tau}, z_{\tau}\right) \\
\text { s.t. } & x_{\tau} \in \mathcal{A}_{z_{\tau}} \text { for } \tau \in\{1,2, \ldots, t\} \\
& \sum_{\tau=1}^{t} x_{\tau} \leq \Delta .
\end{aligned}
$$

At the beginning of the prediction round $n \in\{0,1, \ldots, N-1\}$, which is time slot $n w+1$, the system controller under $C P A(\pi)$ solves the following convex optimization problem: 


$$
\begin{aligned}
\min _{x_{\tau}, t \in \mathcal{T}_{n}} & \sum_{t=n w+1}^{n w+w} x_{t} \\
\text { s.t. } & \sum_{t=n w+1}^{n w+w} R\left(x_{t}, z_{t}\right) \geq \frac{1}{\pi}\left(\eta^{*}(n w+w)-\eta^{*}(n w)\right) \\
& x_{t} \in \mathcal{A}_{z_{t}} \text { for } t \in \mathcal{T}_{n} .
\end{aligned}
$$

The optimal solution of $\mathrm{P} 4$ is denoted by $x_{t}^{\pi}, t \in \mathcal{T}_{n}$. After solving P4, $C P A(\pi)$ makes the decision for time slot $t \in \mathcal{T}_{n}$ as $x_{t}^{\pi}$. Let $\eta^{\pi}$ be the total revenue of P1 under $C P A(\pi)$, i.e., $\eta^{\pi}=\sum_{t=1}^{T} R\left(x_{t}^{\pi}, z_{t}\right)$.

Essentially, $C P A$ tries to approximately track the optimal solution's revenue trajectory round by round via P3. It then uses $\mathrm{P} 4$ to use the least amount of resource possible to match that revenue trajectory. However, notice that the optimal solution to P3 does not match with the optimal solution to $\mathrm{P} 1$ until $t=T$, which means that the revenue trajectory created by $\mathrm{P} 3$ from round to round is only an estimate of the true optimal revenue trajectory. Note that the first constraint in P4 is tight for any optimal solution, i.e., $\sum_{\tau=n w+1}^{n w+w} R\left(x_{\tau}^{\pi}, z_{\tau}\right)=\frac{1}{\pi}\left(\eta^{*}(n w+w)-\eta^{*}(n w)\right)$. Otherwise, if $\sum_{\tau=n w+1}^{n w+w} R\left(x_{\tau}^{\pi}, z_{\tau}\right)>\frac{1}{\pi}\left(\eta^{*}(n w+w)-\eta^{*}(n w)\right)$, then a better solution can be made by decreasing any nonzero $x_{t}^{\pi}, t \in \mathcal{T}_{n}$. Also, note that P4 always has a feasible solution when $\pi>1$.

As in $[15,23]$, we use the competitive ratio to evaluate the performance of $C P A$. Let $A L G$ be a deterministic online algorithm for $\mathrm{P} 1$. ALG is called $\pi$-competitive if

$$
\pi=\max _{\mathbf{z} \in \mathbf{Z}} \frac{\eta^{*}}{\eta^{A}}
$$

where $\eta^{*}$ and $\eta^{A}$ are the revenues of P1 under the optimal offline solution and under $A L G$, respectively.

$C P A(\pi)$ is considered feasible for $\mathrm{P} 1$ only if the total resource consumption under $C P A(\pi)$ does not violate the resource constraint for all possible states $\mathrm{z} \in \mathrm{Z}$ : $\max _{\mathbf{z} \in \mathbf{Z}} \sum_{t=1}^{T} x_{t}^{\pi} \leq \Delta$.

If the decisions chosen by $C P A(\pi)$ are feasible, we have from the first constraint of $\mathrm{P} 4$ that $\sum_{t=1}^{T} R\left(x_{t}^{\pi}, z_{t}\right)=\sum_{n=0}^{N-1} \frac{1}{\pi}\left(\eta^{*}(n w+w)-\eta^{*}(n w)\right)=\frac{1}{\pi} \eta^{*}(T)$. According to the definition of $\eta^{*}(t)$, $\eta^{*}(T)=\eta^{*}$ is the optimal offline revenue of $\mathrm{P} 1$. Thus, if $C P A(\pi)$ is feasible, then

$$
\max _{\mathrm{z} \in \mathrm{Z}} \frac{\eta^{*}}{\eta^{\pi}}=\pi
$$

That is, for any $\pi \geq 1$, as long as $C P A(\pi)$ is feasible, $C P A(\pi)$ is $\pi$-competitive.

We now analyze the performance of $C P A$ by finding the region of $\pi$ such that $C P A(\pi)$ is feasible for P1 in the following theorem.

Theorem 4.2. For any $\pi \geq c\left(1+(T / w-1)\left(1-\theta^{-1 /(T / w-1)}\right)\right)$ where $c=\max _{\mathbf{z} \in \mathbf{Z}} \frac{p(t)}{R\left(\delta_{z_{t}}, z_{t}\right) / \delta_{z_{t}}}$, $C P A(\pi)$ is feasible and $\pi$-competitive for $P 1$.

The proof is found in Appendix B. The proof idea of Theorem 4.2 is as follows. We first generate another set of revenue functions and prove that they have specific properties in Appendix B.1. Then, in Appendix B.2, we define another online optimization problem P8 based on the newly defined revenue functions and propose an algorithm called $C P A 1(\pi)$ to solve P8. Then, in Appendix B.2, we show that for a given $\pi$, if $C P A 1(\pi)$ is feasible for P8, $C P A(\pi)$ will be feasible for P1. Next, in Appendix B.3, we show that for a given $\pi$, if $C R$-Pursuit $(\pi)$ is feasible for P8, then $C P A 1(\pi)$ will be feasible for P8. That is, for a given $\pi$, if $C R$-Pursuit $(\pi)$ is feasible for P8, then $C P A(\pi)$ will be feasible for P1. Then, we show that Lemma 7, Lemma 8 (from [23]), and Lemma 9 hold, which are in Appendices B.4, B.4, and B.5, respectively. After that, in Appendix B.5, we show that for 
$\pi \geq c\left(1+(T / w-1)\left(1-\theta^{-1 /(T / w-1)}\right)\right)$, CR-Pursuit $(\pi)$ is feasible for P8. Finally, in Appendix B.6, for $\pi \geq c\left(1+(T / w-1)\left(1-\theta^{-1 /(T / w-1)}\right)\right), C P A(\pi)$ will be feasible for P1.

According to [23], CR-Pursuit is $c(1+\log (\theta))$-competitive for P1. In Theorem 4.2, we showed that $C P A$ is $c \cdot\left(1+(T / w-1)\left(1-\theta^{-1 /(T / w-1)}\right)\right)$-competitive for P1. Because $\theta^{x}>1+x \cdot \log (\theta)$ for $\theta>0$, we have $\left(1-\theta^{-1 /(T / w-1)}\right)<\left(1+\frac{\log (\theta)}{T / w-1}-1\right)=\frac{\log (\theta)}{T / w-1}$ for $T>w$. Therefore, $(T / w-1)\left(1-\theta^{-1 /(T / w-1)}\right)<$ $\log (\theta)$. That is, by exploiting predictions, CPA has better performance than the CR-Pursuit in terms of competitive ratio.

Because the derivative of $x\left(1-\theta^{-1 / x}\right)$ is less than 0 for $x>0$ and $\theta>1$, as prediction window $w$ increases, $(T / w-1)\left(1-\theta^{-1 /(T / w-1)}\right)$ decreases. This means that as expected, increasing the prediction window size improves the performance of $C P A$. Also, it means that $C P A$ has better performance for systems with shorter time horizon $T$.

\section{META-ALGORITHM}

It is common in many real-world systems that the evolution of system states may not be a fully stationary process nor a fully arbitrarily determined process, but something in between. In fact, where a system lies between these two extremes may also change with time, either gradually or abruptly.

The performance guarantees proved in the previous section assume that it is known which extreme environment the system is operating in beforehand, or when it will switch to the other one and for how long. In this section, we relax this assumption but still try to utilize the power of the previously described online algorithms along with any of the others described in Section 1. We do this by designing a practical meta-algorithm to choose which online algorithm's decision at each time slot and change with a possibly time varying mix of state evolution environments. In doing so, our goal is still to maximize the total revenue gained by the system while respecting the inventory constraint.

Our meta-algorithm makes a performance prediction at each time slot for each online algorithm from the set of algorithms $\Gamma$ and implements the decision from the algorithm with the highest performance prediction. The performance prediction for an algorithm is made from observing how much reward it earned and inventory it used in the most recent $v$ time slots in the past.

The key challenge in designing the meta-algorithm is to determine a reasonable performance metric that can be used to compare algorithms that earn different amounts of revenue and use varying amounts of their inventories. Simply comparing earned revenues directly, such as in the Follow the Leader meta-algorithm, leaves out the fact that each algorithm may have used different amounts of their inventory. Thus, each leaves more or less inventory available for the future, which makes their revenues incomparable. On the other hand, we could evaluate the performance of an algorithm by looking at its efficient use of the inventory, the ratio of revenue earned to inventory used, in the past. However, this value also cannot be directly compared between algorithms as a predictor of performance since it says nothing about the rate at which revenue is being earned and how much inventory remains. It is possible that a highly efficient algorithm may only use a very little portion of its inventory, thus resulting in a small amount of revenue earned. A more inefficient algorithm may have earned more revenue by utilizing more of its inventory.

Instead, for the most recent $v$ time slots in the past, we look at each algorithm's average rate of earning revenue along with its average rate of consuming inventory. If we extrapolate its average inventory consumption rate into the future, the algorithm will either (1) run out of the meta-algorithm's inventory, (2) run out of its own inventory, or (3) not utilize all of its inventory before reaching the time horizon $T$. Assuming that its ratio of revenue earned to inventory 


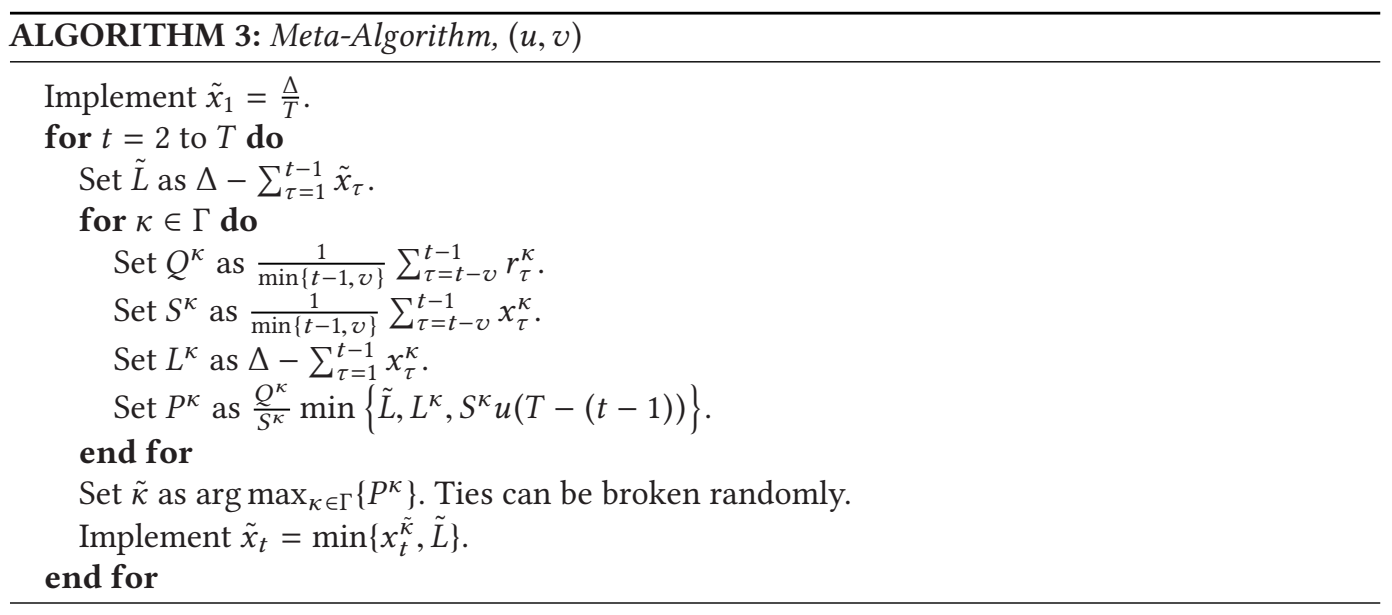

consumed on average remains constant, its future revenue earnings will be limited by either the remaining inventory in cases (1) and (2) or the remaining number of time slots in case (3). Thus, we can calculate each algorithm's future potential revenue, limited by either inventory or time, and use this as the performance metric for deciding which algorithm's decision to implement since they are directly comparable between algorithms.

The details of the meta-algorithm are given in Algorithm 3. At each time slot $t$ and looking into $v$ time slots of the most recent past, it calculates for each online algorithm $\kappa$ the average rate of earning revenue $Q^{\kappa}$ and the average rate of consuming inventory $S^{\kappa}$, and also calculates the remaining inventory $L^{\kappa}$. Afterward, it calculates its future potential revenue $P^{\kappa}$ by multiplying its ratio of revenue earned to inventory consumed $\frac{Q^{\kappa}}{S^{\kappa}}$ by either (1) the remaining inventory of the meta-algorithm $\tilde{L},(2)$ its own remaining inventory $L^{\kappa}$, or (3) its inventory to be used in the remaining $T-(t-1)$ time slots discounted by a parameter $u$ at an assumed consumption rate of $S^{\kappa}$, whichever case is smaller. Finally, the meta-algorithm chooses the algorithm $\tilde{\kappa}$ that has the highest predicted future earnings and implements the decision $x_{t}^{\tilde{\kappa}}$ upper bounded by the remaining inventory of the meta-algorithm $\tilde{L}$ to make sure the inventory condition is satisfied.

The parameter $u \in[0,1]$ is used to discount the amount of remaining time slots used in the calculation of an algorithm's future potential revenue $P^{\kappa}$. Decreasing $u$ lowers the calculated future potential revenue for algorithms that conserve inventory, but it does not affect algorithms that consumed larger amounts of inventory in the past. Essentially, a value of $u$ closer to 0 promotes algorithms that consume the inventory quickly, while a value of $u$ closer to 1 promotes algorithms that conserve inventory. The best value of $u$ will depend on how quickly the controller should be consuming inventory.

The parameter $v \in\{1, \ldots, T\}$ in the meta-algorithm determines how many recent time slots are used to make its performance prediction. For a system that either rarely switches between stationary and arbitrary state evolution environments or switches gradually, $v$ should be set to a high value to get an accurate future potential revenue calculation. On the other hand, if the system switches between environments often and abruptly, then $v$ should be set to a low value so that the performance prediction can adapt quickly. Also, we can choose parameters based on their previous performance. To be more specific, we can apply the meta-algorithm under different parameters in parallel to the system using previous inputs of the system and choose the parameters with the best performance. This is similar to "follow the leader" idea in machine learning. There are also other methods to choose parameters, e.g., reinforcement learning. We leave them for future work. 


\section{PERFORMANCE ANALYSIS}

In this section, we evaluate the performance of our online algorithms from Section 4 and the metaalgorithm from Section 5 under different system state environments in the application setup of collecting data of shared bikes.

\subsection{Setup}

There is a company operating a dockless bike sharing system [12] over $T$ time slots, i.e., $\{1,2, \ldots, T\}$. Each time slot can be several minutes. There are some base stations located in different places of the city to receive data from shared bikes.

Each bike of the system moves by the corresponding renter over a city. There is a sensor with a battery of size $\Delta$ in each bike that collects data, e.g., location of the bike, speed of the bike, and road conditions, and sends data to the nearest base station. The batteries of each bike are fully recharged by its operator at the beginning. Since all shared bikes are operated independently, we focus on one bike. The sensor of the bike uses a wireless channel of bandwidth $W$ to transmit data. At each time slot $t$, the sensor of the bike transmits data to the nearest base station by consuming energy of size $x_{t}$ from the battery. The goal is to transmit as much data as possible to base stations. $z_{t}$, the signal attenuation rate between the sensor and its nearest base station at time slot $t$, is the system state and varies with time. The noise power of the channel is $N$, which is known in advance. The data rate at time slot $t$, denoted by $R\left(x_{t}, z_{t}\right)$, can be calculated as

$$
R\left(x_{t}, z_{t}\right)=W \cdot \log \left(1+\frac{z_{t} \cdot x_{t}}{N}\right),
$$

where $x_{t}$ is the amount of energy used for the current time slot from the limited battery.

At the beginning of each time slot, the sensor is provided with predictions of attenuation rates during the next $w$ slots. This is reasonable as the attenuation rate can be modeled as a function of the weather $[2,3,6,10,21,28,37]$, e.g., rain fall rate and fog density, and the weather can be predicted with high accuracy in the short term, and the distance to the nearest base station can be predicted [13]. With the predictions available, the system controller needs to decide $x_{t}$ for the goal of maximizing the total data transferred to base stations over the time horizon while respecting the constraint from the battery $\sum_{t} x_{t} \leq \Delta$ for total energy usage.

Specifically, we set the bandwidth $W=200 \mathrm{MHz}$ and the noise power $N=-50 \mathrm{dBw} / \mathrm{Hz}$. The battery size $\Delta$ is 3,000 $\mathrm{mWh}$ and the power of each time slot cannot exceed $10 \mathrm{~W}$. Each time slot lasts for 1 minute in the simulations. For most of the simulations presented, unless stated otherwise, we mix the attenuation rate traces shown in Figure 1 with a mixing parameter $\lambda \in[0,1]$ at time slot $t$ :

$$
z_{t}^{(\lambda)}=\lambda z_{t}^{(a)}+(1-\lambda) z_{t}^{(b)}
$$

where $z_{t}^{(a)}$ and $z_{t}^{(a)}$ come from the traces in Figures 1(a) and 1(b), respectively. With the use of the mixing parameter $\lambda$, we can modulate between the scenarios described in Section 2.

Since meta-algorithms choose their actions based on the options created by individual algorithms, we run several algorithms in parallel under various settings when applicable: $C P A$ with $\pi=\left\{1, \frac{\pi^{*}}{5}, \frac{\pi^{*}}{6}, \ldots, \frac{\pi^{*}}{11}\right\}$, where $\pi^{*}$ is the minimum value of $\pi$ that satisfies Theorem 4.2; CPS; RHC; $F H C$; and $E D$, which is the naive algorithm that simply distributes the inventory equally among the time slots, i.e., $x_{t}^{E D}=\frac{\Delta}{T}$. Because $R H C$ and $F H C$ were not specifically designed for solving problem $\mathrm{P} 1$, we add the following constraint to their individual optimization problems to handle the inventory constraint: $\sum_{\tau=t}^{t+w-1} x_{\tau} \leq w \frac{\Delta}{T}$, which assumes that the inventory can be distributed relatively equally. All algorithms are run with a prediction window size $w$ of 5 unless noted otherwise. 


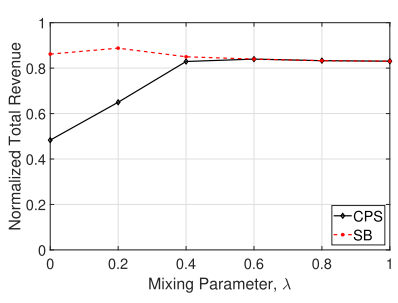

(a) CPS

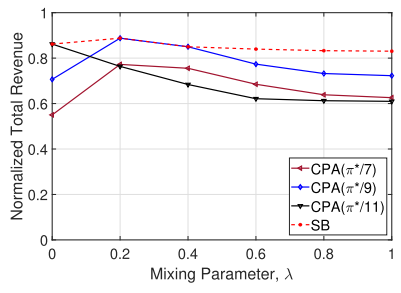

(b) CPA

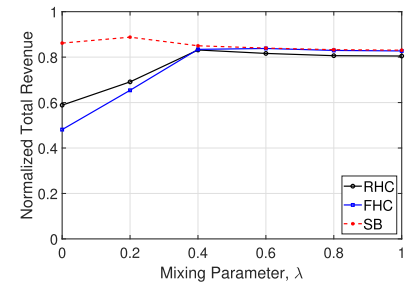

(c) $\mathrm{RHC} / \mathrm{FHC}$

Fig. 2. Normalized total revenue vs. mixing parameter $\lambda$ to modulate between the scenario with increasing attenuation rates and spiking attenuation rates for online algorithms.

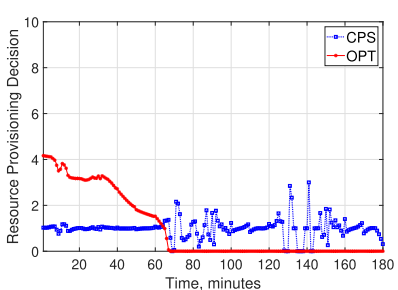

(a) CPS

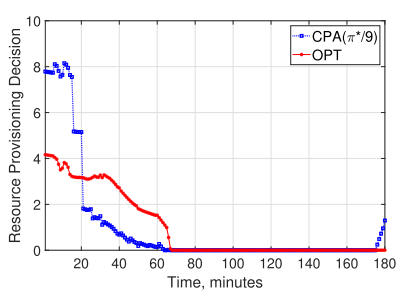

(b) CPA

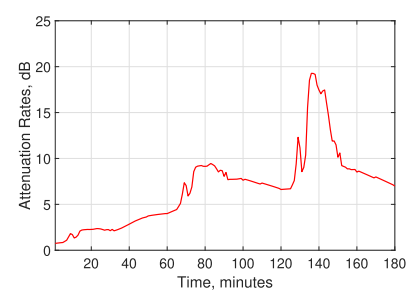

(c) Attenuation Rate

Fig. 3. Control actions for (a) CPS and (b) CPA compared to the optimal solution under the scenario $\lambda=0.2$, which gives the attenuation rate trace in (c).

When comparing the total earned revenue for each algorithm under different scenarios, we compare each of them against the Single Best $(\boldsymbol{S B})$ algorithm, which can be thought of as an idealistic meta-algorithm. That means for any specific system state environment and among all of the algorithms ran in parallel, in hindsight $S B$ will pick the single one that earned the most revenue. Additionally, we compare our meta-algorithm against the Follow the Leader (FTL), which just chooses the each time slot that currently has earned the most revenue so far.

\subsection{Results}

6.2.1 Online Algorithms. We evaluate the online algorithms designed in Section 4 and ones existing in the literature under different scenarios of system state environments. Using the mixing parameter $\lambda$, we are able to go between a scenario with an increasing attenuation rate (Figure 1(a)) at $\lambda=0$ and a scenario with spikes (Figure $1(\mathrm{~b})$ ) at $\lambda=1$. The results can be seen in Figure 2 .

CPS. Since CPS was designed for a stationary environment, from Figure 2(a), we can see that it does well for the scenario when the attenuation rate has the pattern of repeating spikes $(\lambda \in$ $\{0.4,0.6,0.8,1\})$ and even matches the Single Best algorithm. From Figure 4(a), we can see that the control actions are relatively close to that of the optimal solution compared to CPA for $\lambda=$ 0.6 . However for the increasing scenario $(\lambda \in\{0,0.2\})$, it does poorly because it distributes the inventory relatively equally across the operating time, whereas the optimal solution uses most of the inventory in the first third of the operating time to take advantage of the low attenuation rates (see Figure 3(a)). Observe that from Figure 2(c), RHC and FHC have the same behavior of performance for the same reasons. While CPS, RHC, and FHC show similar performance in these simulations, $C P S$ comes with the additional feature of having performance guarantees as shown in Section 4.1. 


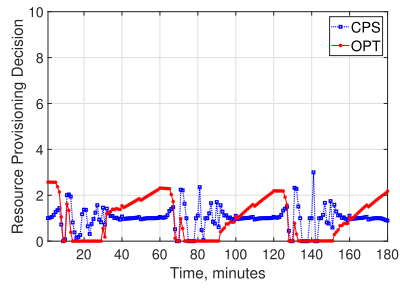

(a) CPS

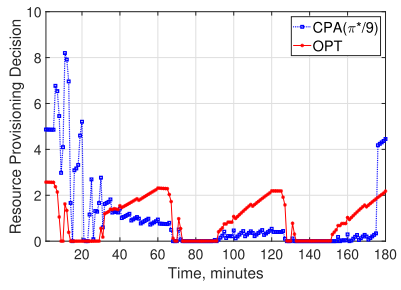

(b) CPA

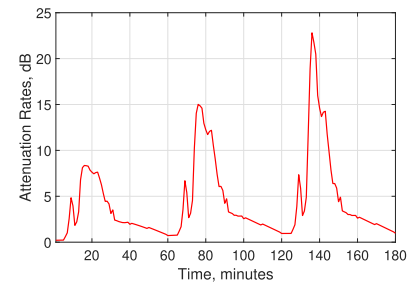

(c) Attenuation Rate

Fig. 4. Control actions for (a) CPS and (b) CPA compared to the optimal solution under the scenario $\lambda=0.6$, which gives the attenuation rate trace in (c).

$C P A$. Under the pessimistic assumption of an environment where states can be arbitrarily determined, even adversarially determined, the value of $C P A$ 's parameter $\pi$ proved in Theorem 4.2 that makes it feasible in all situations is quite high for a practical scenario. Thus, we label the minimum feasible $\pi$ according to Theorem 4.2 as $\pi^{*}$ and set $\pi$ in the simulations as fractions of that. We can observe in Figure 2(b) that $C P A$ can do well in the increasing attenuation rate environments $(\lambda \in\{0,0.2,0.4\}$ under different settings of $\pi$. This is because as $\pi$ decreases, CPA gets more aggressive with its consumption of the inventory in the beginning of the operating time. Thus, at $\lambda=0$, the setting $\pi=\frac{\pi^{*}}{11}$ closely tracks the optimal solution that uses inventory heavily in the beginning. However as $\lambda$ increases to 0.2 or 0.4 , the optimal solution pushes the inventory concentration away from the beginning, which makes the setting $\pi=\frac{\pi^{*}}{9}$ match the Single Best algorithm. This can be specifically shown by comparing Figures 3(b) and 4(b), which are the control actions of $C P A\left(\pi^{*} / 9\right)$ under scenarios $\lambda=0.2$ and $\lambda=0.6$, respectively. In the first scenario, $\lambda=0.2, C P A^{\prime}$ s behavior closely matches that of the optimal solution by concentrating inventory consumption near the beginning. However, in the second scenario $\lambda=0.6, C P A$ still concentrates most of its inventory consumption in the beginning, whereas the optimal solution does not.

6.2.2 Meta-Algorithm. We evaluate our meta-algorithm under two settings against SB and FTL under various scenarios of system state environments. The parameter $u$ is made to have two separate settings $\{0.1,0.7\}$. The lower the value of $u$, the more the meta-algorithm gives priority to algorithms that earn revenue rapidly over ones that conserve inventory for the future. The parameter $v$ of our meta-algorithm is set to be 20 , which means that it uses the 20 previous time slots to make its prediction of which algorithm will have the most future earnings among all that are run in parallel.

Figure 6 shows the performance of our meta-algorithm under different scenario mixes. It significantly outperforms $F T L$, and depending on its setting of $u$, it consistently performs better than the Single Best algorithm chosen in hindsight. Specifically, when $u$ is set to 0.1 , it performs better for $\lambda \in\{0,0.2\}$, and when $u$ is set to 0.7 , it performs better for $\lambda \in\{0.4,0.6,0.8,1\}$. The sensitivity of the meta-algorithm's performance with respect to $u$ under different scenarios is shown in Figure 5(a). We can see that for $\lambda=0.2$, the meta-algorithm's performance decreases as $u$ increases from 0.1 due to a larger $u$ prioritizing algorithms that use its inventory conservatively. For $\lambda=0.6$, the performance decreases as $u$ decreases from 0.7 due to a smaller $u$ prioritizing algorithms that use its inventory aggressively. From Figures 5(b) and 5(c), we can see that when $u$ is set appropriately, the meta-algorithm will make decisions that match the behavior of the optimal solution, even closer than that of CPS and CPA in Figures 3 and 4. Notice that a proper setting of $u$ makes the meta-algorithm use up its inventory around the same time that the optimal solution does. 


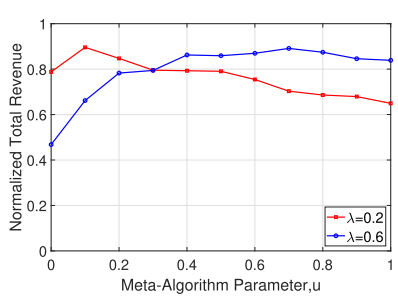

(a) Parameter $u$

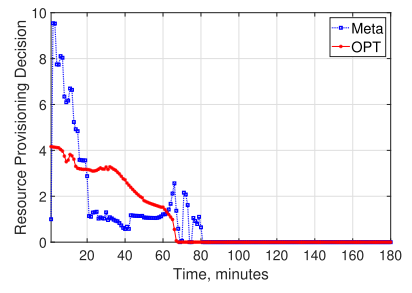

(b) Control Actions: $\lambda=0.2, u=0.1(c)$

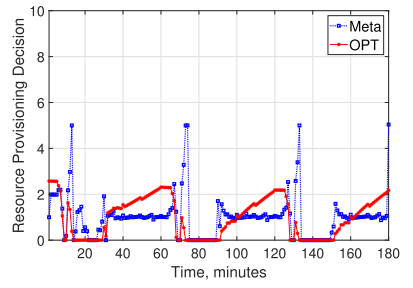

(c) Control Actions: $\lambda=0.6, u=0.7$

Fig. 5. (a) Meta-algorithm's total revenue earned vs. (b) the meta-algorithm's parameter $u$ under different attenuation rate scenarios $\lambda \in\{0.2,0.6\}$. All revenues are normalized by the optimal value for a given $\lambda$. Control actions of the meta-algorithm vs. the optimal solution for (b) $\lambda=0.2, u=0.1$ and (c) $\lambda=0.6$, $u=0.7$.

6.2.3 Prediction Errors. Up to this point, we have assumed that the predictions given to the online algorithms are perfect within their prediction window. In this set of simulations we relax this assumption by adding error to the predictions. We create our trace of prediction errors based on the usage of Naive Predictions. At time slot $t$, Naive Predictions are created by using the state at time slot $t-1$ as the predictions for all states from time slots $t$ to $t+w-1$. From these naive predictions, we calculate their errors from the true state values. This set of errors becomes the error trace that we add to the perfect predictions to make them imperfect. If we just add these errors directly, we obtain the Naive Predictions, which are very pessimistic. We use the parameter $\kappa \in[0,1]$ to modulate between perfect predictions with no error $(\kappa=0)$ and Naive Predictions with high error $(\kappa=1)$. From Figure 7, which shows the total earned revenue, we can see that CPS and CPA remain relatively flat when $\kappa$ is increased from 0 to 1 , and thus are shown to be robust to prediction error.

6.2.4 Prediction Window Size Effects. Here we investigate how the prediction window size $w$ affects the performance of the online algorithms described in Section 4 in system environments for which they were designed.

CPS with stationary attenuation rates. We simulate the system environment using i.i.d attenuation rates $z_{t}$ drawn from a Gaussian distribution with an expectation of 0.2 and a standard deviation of 0.04 . The prediction window size is varied $w=\{1,2,4,8\}$ and the time horizon $T$ is set to 200 .

We compare the performance of CPS with those of the optimal offline solution and CR-Pursuit. As shown in Figure 8, the total amount of data transmitted under CPS is near optimal, even with prediction window size of 1 , and the total data transmitted under CPS increases as the prediction window $w$ size increases. However, the total data transmitted by CR-Pursuit is only $13 \%$ of that of $C P S$ with a prediction window size $w$ of 1 .

CPA with non-stationary attenuation rates. We simulate the system environment using a real trace of measured attenuation rates with carrier frequency of $40 \mathrm{GHz}$ [28] repeated three times (see Figure 1(b)). We set the prediction window size to $w=\{4,5,6,10\}$ and time horizon $T$ to 60 .

We compare the results of $C P A$ with those of $C R$-Pursuit and the optimal offline solution. As shown in Figure 9, the total data transmitted under CPA is better than that under CR-Pursuit. Again, the total data transmitted under $C P A$ increases as the prediction window size increases. The gap between $C P A$ and the optimal solution is larger compared to Figure 8 . This is because the predictions within the prediction window don't necessarily reveal more information about the system as is the case for the stationary environment. 


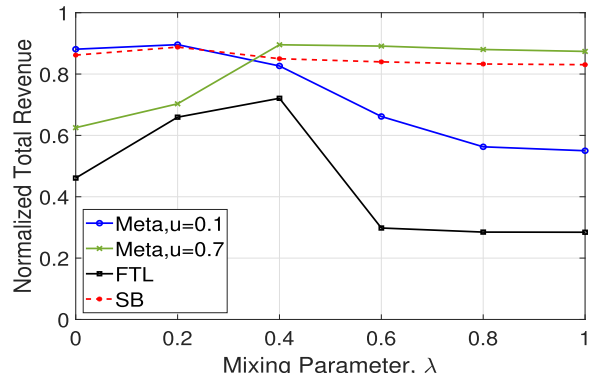

Fig. 6. Meta-algorithm's total revenue earned vs. different scenarios via the mixing parameter $\lambda$ compared to $S B$ and $F T L$.

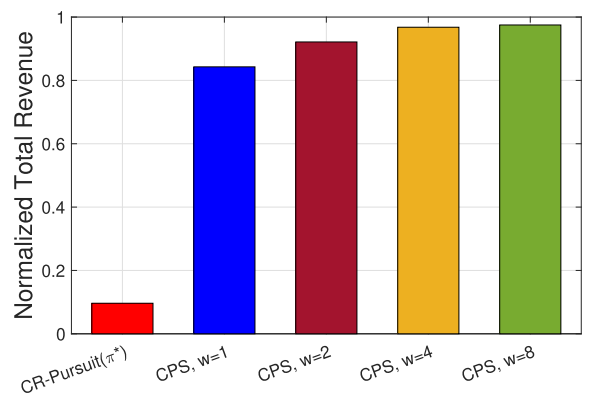

Fig. 8. Comparison of the amount of total data transmitted between CR-Pursuit and CPS under i.i.d. attenuation rates.

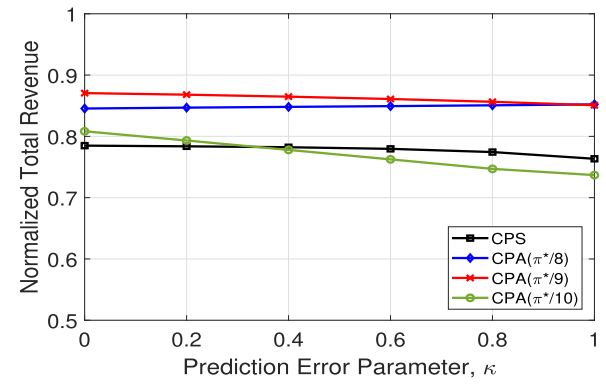

Fig. 7. Normalized total revenue vs. prediction error parameter $\kappa$ for the online algorithms CPS and CPA with different parameters in scenario $\lambda=0.5$.

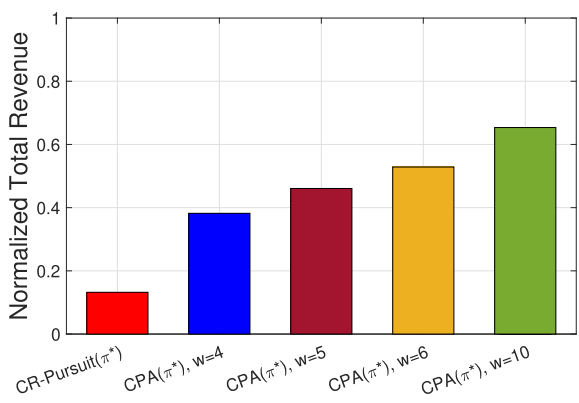

Fig. 9. Comparison of the amount of total data transmitted between CR-Pursuit and CPA under non-stationary attenuation rates.

\section{RELATED WORK}

Online optimization problems in general have a rich history and have been implemented in various applications $[1,7,15,23]$. There are different versions of online problems, e.g., the one-way trading problem $[11,15,36]$, the secretary problem, and prophet inequalities $[5,16,31]$. Some online problems like ours focus on the situation in which the control actions are subject to an inventory constraint $[4,23]$. The problem we consider in this article is a generalization of the one-way trading problem [15] and the online optimization under inventory constraints (OOIC) [23].

The application of online resource provisioning that we simulate is the data collection of shared bikes using wireless sensors. Bike sharing has become a new urban lifestyle recently that facilitates people's daily life and has expanded quickly $[12,30]$. To provide better service, a service provider collects data of shared bikes and uses data to optimize their operation for different purposes [14, $24,38]$. To collect real-time data of shared bikes, sensors located in shared bikes need to transmit as much data as possible to base stations during its operating time horizon with the constraint that the total energy consumed is less than its battery size.

\section{CONCLUSION}

In this article, we consider the online resource provisioning problem with short-term predictions, which can be applied to a large number of real-world applications including collecting data of shared bikes of a bike sharing system. The way an algorithm uses the predictions depends on how the system environment behaves. Thus, we develop two online algorithms and prove their 
performance, each designed for a different system environment type. For stationary environments, we prove that the algorithm CPS has a lower bound on expected performance that is independent of $\theta$, which is the ratio between the maximum and minimum gradients of the revenue with no resource being used. For arbitrarily determined environments, we prove that the algorithm $C P A$ has a lower bound on the Competitive Ratio, which depends on $\theta$ but decreases as the prediction window size increases. We also propose a meta-algorithm that evaluates how much revenue each algorithm is earning with respect to the amount of resources it has used so far and implements the resource provisioning decision from the one it predicts will earn the most in the future We evaluate the online algorithms and the meta-algorithm with trace-driven simulations under different scenarios in the application of collecting data of shared bikes. In the application, sensors equipped in shared bikes need to transmit data to base stations under time-varying attenuation rates. The goal is to maximize the total data transmitted over the operating time horizon with the constraint that the total energy consumed is less than the battery size. Simulation results show that by adjusting a single parameter, the meta-algorithm can outperform the single best algorithm in hindsight in different environments.

\section{APPENDICES}

\section{A PERFORMANCE ANALYSIS FOR CPS}

The predictions can be used to calculate the relative frequencies of each state within the prediction window. At time slot $t$, the relative frequency of $z(i)$ over the current prediction window is $\sum_{\tau=t}^{t+w-1} \mathbb{1}_{i}\left(z_{\tau}\right) / w$, where $\mathbb{1}_{i}:\{z(1), z(2), \ldots, z(I)\} \rightarrow\{0,1\}$ is an indicator function that determines $\mathbb{1}_{i}\left(z_{t}\right)=1$ if $z_{t}=z(i)$, and $\mathbb{1}_{i}\left(z_{t}\right)=0$ otherwise. We denote $\mathbf{f}=\left(f_{1}, f_{2}, \ldots, f_{I}\right)$ as the relative state frequencies for each state over a specified time interval $\hat{\mathcal{T}}$, i.e., $f_{i}=\frac{\sum_{\tau \in \hat{\mathcal{T}}} \mathbb{1}_{i}\left(z_{\tau}\right)}{|\hat{\mathcal{T}}|}$.

\section{A.1 Lemma 1}

We begin with Lemma 1, which measures the difference between the relative frequencies of system states in a prediction window and the real probability mass function of system states.

According to the Chernoff bound, we have $\operatorname{Pr}\left(\left|\frac{\sum_{\tau t}^{t+w-1} \mathbb{1}_{i}\left(z_{\tau}\right)}{w}-p_{i}\right| \geq p_{i} \epsilon\right) \leq 2 e^{-\frac{w \epsilon^{2} p_{i}}{3}}$ for $\epsilon \in(0,1)$. Thus, $\operatorname{Pr}\left(\bigcup_{i \in[I]}\left\{\left|\frac{\sum_{\tau=t}^{t+w-1} \mathbb{1}_{i}\left(z_{\tau}\right)}{w}-p_{i}\right| \geq p_{i} \epsilon\right\}\right) \leq \sum_{i \in[I]} \operatorname{Pr}\left(\left|\frac{\sum_{\tau=t}^{t+w-1} \mathbb{1}_{i}\left(z_{\tau}\right)}{w}-p_{i}\right| \geq p_{i} \epsilon\right) \leq \sum_{i \in[I]} 2 e^{-\frac{w \epsilon^{2} p_{i}}{3}}$ for all $\epsilon \in(0,1)$. We state it in Lemma 1 as follows.

Lemma 1. For P1 with system state probability distribution $\mathbf{p}=\left(p_{1}, p_{2}, \ldots, p_{I}\right)$, at an arbitrary time slot $t$, we have $\operatorname{Pr}\left(\bigcup_{i \in[I]}\left\{\left|\frac{\sum_{\tau=t}^{t+w-1} \mathbb{1}_{i}\left(z_{\tau}\right)}{w}-p_{i}\right| \geq p_{i} \epsilon\right\}\right) \leq \sum_{i=1}^{I} 2 e^{-\frac{w \epsilon^{2} p_{i}}{3}}$ for $\forall \epsilon \in(0,1)$.

Here, $\sum_{\tau=t}^{t+w-1} \mathbb{1}_{i}\left(z_{\tau}\right) / w$ is the relative frequency of $z(i)$ over prediction window $\{t, t+1, \ldots, t+$ $w-1$. Lemma 1 shows that given a window size $w$ and any $\epsilon \in(0,1)$, the probability of $\exists i$ such that $\left|\sum_{\tau=t}^{t+w-1} \mathbb{1}_{i}\left(z_{\tau}\right) / w-p_{i}\right| \geq p_{i} \epsilon$ is no greater than $\sum_{i=1}^{I} 2 e^{-w \epsilon^{2} p_{i} / 3}$. As $w$ increases, the probability of $\exists i$ such that $\left|\sum_{\tau=t}^{t+w-1} \mathbb{1}_{i}\left(z_{\tau}\right) / w-p_{i}\right| \geq p_{i} \epsilon$ decreases exponentially.

\section{A.2 Lemma 2}

Then, we introduce Lemma 2, which measures the difference between the expectation of the optimal objective value of $\mathrm{P} 1$ with system state probability distribution $\mathbf{p}=\left(p_{1}, p_{2}, \ldots, p_{I}\right)$ and the optimal objective value of $\mathrm{P} 2$ with relative state frequencies $\mathbf{f}=\left(f_{1}, f_{2}, \ldots, f_{I}\right)$ over $\tau \in\{t, t+1, \ldots, t+w-1\}$. For $\mathrm{P} 1$ with system state probability distribution $\mathbf{p}$, states are randomly drawn from a multinoulli distribution with probabilities $\left(p_{1}, p_{2}, \ldots, p_{I}\right)$. For P2 with relative state frequencies $\mathbf{f}=\left(f_{1}, f_{2}, \ldots, f_{I}\right)$, the relative frequency of $z(i)$ over $\tau \in\{t, t+1, \ldots, t+w-1\}$ is $f_{i}$. $\mathbb{E}\left[\eta^{*}\right]$ denotes the expectation of the optimal objective value of $\mathrm{P} 1$ with system state probability 
distribution $\mathbf{p}=\left(p_{1}, p_{2}, \ldots, p_{I}\right)$ and uses $\eta_{\mathrm{f}}^{*}$ to denote the optimal objective values of $\mathrm{P} 2$ with relative state frequencies $\mathbf{f}=\left(f_{1}, f_{2}, \ldots, f_{I}\right)$.

Lemma 2. For $P 1$ with system state probability distribution $\mathbf{p}=\left(p_{1}, p_{2}, \ldots, p_{I}\right)$ and $P 2$ with relative state frequencies $\mathbf{f}=\left(f_{1}, f_{2}, \ldots, f_{I}\right)$, given $\epsilon \in(0,1)$, if $\left|f_{i}-p_{i}\right| \leq \epsilon p_{i}$ holds for all $i \in[I]$, then we have $\frac{1}{w} \eta_{\mathrm{f}}^{*} \geq \frac{1}{T} \mathbb{E}\left[\eta^{*}\right] \frac{(1-\epsilon)}{1+\epsilon}$.

Proof. Use $x_{t \mid \tau}, \tau \in t, t+1, \ldots, t+w-1$ to represent the optimal solution of P2. For any $i \in$ [I], from Jensen's inequality, if $x_{t \mid \tau}, \tau \in\left\{t, t+1, \ldots, t+\tau-1 \mid z_{\tau}=z(i)\right\}$ are not equal, we can have another optimal solution by replacing $x_{t \mid \tau}, \tau \in\left\{t, t+1, t+\tau-1 \mid z_{\tau}=z(i)\right\}$ by the mean of them. Therefore, there exists an optimal solution of P2 where $x_{t \mid \tau}$ and $x_{t \mid \tau^{\prime}}$ are equal if $z_{\tau}=z_{\tau^{\prime}}$. Thus, the optimal value of $\mathrm{P} 2$ is equal to the optimal objective value of the following optimization problem:

$$
\begin{array}{ll}
\max _{x_{i}} & w \sum_{i=1}^{I} f_{i} \cdot R\left(x_{i}, z_{i}\right) \\
\text { s.t. } & x_{i} \in \mathcal{A}_{z_{i}} \text { for } i \in\{1,2, \ldots, I\} \\
& T \sum_{i=1}^{I} f_{i} x_{i} \leq \Delta .
\end{array}
$$

Similarly, we have that for $\mathrm{P} 1$ with system state probability distribution $\mathbf{p}$, the expectation of the optimal objective value of P1 $\mathbb{E}\left[\eta^{*}\right]$ is no greater than $\eta_{\mathrm{p}}^{*}$, the optimal objective value of P6:

$$
\begin{array}{ll}
\max _{x_{i}} & T \sum_{i} p_{i} \cdot R\left(x_{i}, z_{i}\right) \\
\text { s.t. } & x_{i} \in \mathcal{A}_{z_{i}} \text { for } i \in\{1,2, \ldots, I\} \\
& T \sum_{i} p_{i} x_{i} \leq \Delta .
\end{array}
$$

The proof of the above statement equivalence is omitted due to space limitations. Assume $x_{i}^{f}, i \in$ $[I]$, and $x_{i}^{p}, i \in[I]$ are optimal solutions of $\mathrm{P} 5$ and $\mathrm{P} 6$ respectively.

We now prove that if $\left|f_{i}-p_{i}\right| \leq \epsilon p_{i}$, then $\sum_{i=1}^{I} f_{i} R\left(\left(x_{i}^{f}\right), z_{i}\right) \leq \sum_{i=1}^{I} \frac{f_{i}}{1+\epsilon} R\left(\left(x_{i}^{p}\right), z_{i}\right)$. Since $\mid f_{i}-$ $p_{i} \mid \leq \epsilon p_{i}$, we have $p_{i} \geq f_{i} /(1+\epsilon)$. Since $x_{i}^{p}, i \in[I]$ is a solution of $\mathrm{P} 6$, we have $T \sum_{i=1}^{I} p_{i} x_{i}^{p} \leq \Delta$. Since $T \sum_{i=1}^{I} p_{i} x_{i}^{p} \leq \Delta$ and $p_{i} \geq f_{i} /(1+\epsilon)$, we have that $T \sum_{i=1}^{I} f_{i} \frac{x_{i}^{p}}{1+\epsilon} \leq \Delta$. That is, $x_{i}=\frac{x_{i}^{p}}{1+\epsilon}$ is a feasible solution of P5. Since $x_{i}=\frac{x_{i}^{p}}{1+\epsilon}$ is a feasible solution of P5 and $x_{i}^{f}, i \in[I]$ is the optimal solution of $\mathrm{P} 5$, we have that

$$
T \sum_{i=1}^{I} f_{i} R\left(\frac{x_{i}^{p}}{1+\epsilon}, z_{i}\right) \leq T \sum_{i=1}^{I} f_{i} R\left(x_{i}^{f}, z_{i}\right) .
$$

Since $R\left(0, z_{i}\right)=0$ and $R\left(x, z_{i}\right)$ is concave on $x$, we have from Jensen's inequality that $R\left(\frac{x_{i}^{p}}{1+\epsilon}, z_{i}\right) \geq$ $\frac{R}{1+\epsilon}\left(x_{i}^{p}, z_{i}\right)$. From $R\left(\frac{x_{i}^{p}}{1+\epsilon}, z_{i}\right) \geq \frac{R}{1+\epsilon}\left(x_{i}^{p}, z_{i}\right)$ and Equation (1), we have that

$$
T \sum_{i=1}^{I} \frac{f_{i}}{1+\epsilon} R\left(x_{i}^{p}, z_{i}\right) \leq T \sum_{i=1}^{I} f_{i} R\left(x_{i}^{f}, z_{i}\right) .
$$

Since $f_{i} \geq(1-\epsilon) p_{i}$, we have $T \sum_{i=1}^{I} f_{i} R\left(x_{i}^{p}, z_{i}\right) \geq T \sum_{i=1}^{I}(1-\epsilon) p_{i} R\left(x_{i}^{p}, z_{i}\right)$. Combining $T \sum_{i=1}^{I} f_{i} R\left(x_{i}^{p}, z_{i}\right) \geq T \sum_{i=1}^{I}(1-\epsilon) \cdot p_{i} \cdot R\left(x_{i}^{p}, z_{i}\right)$ and Equation (2), we have that $\frac{1-\epsilon}{1+\epsilon} T \sum_{i=1}^{I} p_{i} R\left(x_{i}^{p}, z_{i}\right) \leq T \sum_{i=1}^{I} f_{i} R\left(x_{i}^{f}, z_{i}\right)$. That is, $\frac{1}{w} \eta_{\mathrm{f}}^{*} \geq \frac{1}{T} \eta_{\mathrm{p}}^{*} \cdot \frac{(1-\epsilon)}{1+\epsilon} \geq \frac{1}{T} \mathbb{E}\left[\eta^{*}\right] \cdot \frac{(1-\epsilon)}{1+\epsilon}$. 


\section{A.3 Proof of Theorem 4.1}

Since $R(x, z)$ is concave in $x$ and $x_{t}^{S}=\frac{1}{\min \{t, w\}} \sum_{\tau=\max \{1, t-w+1\}}^{t} x_{\tau \mid t}$, we have from Jensen's inequality that $r_{t}=R\left(x_{t}^{S}, z_{t}\right) \geq \frac{\sum_{\tau=\max \{1, t-w+1\}}^{t} R\left(x_{\tau \mid t}, z_{t}\right)}{\min \{t, w\}}$. Summing the above inequality up for $t \in\{1,2, \ldots, T\}$ yields

$$
\eta^{S}=\sum_{t=1}^{T} r_{t} \geq \sum_{t=1}^{T} \sum_{v=0}^{\min \{t, w\}-1} \frac{1}{\min \{t, w\}} R\left(x_{(t-v) \mid t}, z_{t}\right) .
$$

From Lemma 1, we have

$$
\operatorname{Pr}\left(\bigcup_{i \in[I]}\left\{\left|\frac{\sum_{\tau=t}^{t+w-1} \mathbb{1}_{i}\left(z_{\tau}\right)}{w}-p_{i}\right| \leq p_{i} \epsilon\right\}\right) \geq 1-\sum_{i=1}^{I} 2 e^{-\frac{w \epsilon^{2} p_{i}}{3}}
$$

Since the revenue is no less than 0, combining Equation (4), Lemma 2, and the total law of probability yields

$$
\mathbb{E}\left[\frac{1}{w} \sum_{\tau=t}^{t+w-1} R\left(x_{t \mid \tau}, z_{\tau}\right)\right] \geq \frac{1}{T} \frac{1-\epsilon}{1+\epsilon} \cdot \mathbb{E}\left[\eta^{*}\right] \cdot\left(1-\sum_{i=1}^{I} 2 e^{-\frac{w \epsilon^{2} p_{i}}{3}}\right) .
$$

Since $z_{t}$ are i.i.d over time, the expectations of $R\left(x_{(t-v) \mid t}, z_{t}\right)$ are identical for all $t \in\{1,2, \ldots, T\}$ and $v \in\{0,1, \ldots, \min \{t-1, w-1\}\}$. Since the expectations of $R\left(x_{(t-v) \mid t}, z_{t}\right)$ are identical for all $t \in\{1,2, \ldots, T\}$ and $v \in\{0,1, \ldots, \min \{t-1, w-1\}\}$, from Equation (5), we have

$$
\mathbb{E}\left[R\left(x_{(t-v) \mid t}, z_{t}\right)\right] \geq \frac{1}{T} \frac{1-\epsilon}{1+\epsilon} \cdot \mathbb{E}\left[\eta^{*}\right] \cdot\left(1-\sum_{i=1}^{I} 2 e^{-\frac{w \epsilon^{2} p_{i}}{3}}\right), v \in\{0,1, \ldots, \min \{t-1, w-1\}\} .
$$

From Equations (3) and (6), we have that

$$
\sum_{t=1}^{T} \mathbb{E}\left[r_{t}\right] \geq \sum_{t=1}^{T} \sum_{v=0}^{\min \{t, w\}-1} \frac{\mathbb{E}\left[\eta^{*}\right] \cdot\left(1-\sum_{i=1}^{I} 2 e^{-\frac{w \epsilon^{2} p_{i}}{3}}\right) \frac{1-\epsilon}{1+\epsilon}}{T \cdot \min \{t, w\}}=\mathbb{E}\left[\eta^{*}\right] \cdot \frac{1-\epsilon}{1+\epsilon} \cdot\left(1-\sum_{i=1}^{I} 2 e^{-\frac{w \epsilon^{2} p_{i}}{3}}\right) .
$$

According to CPS, we have $x^{S}(t)=\frac{1}{\max \{w, t+1\}} \sum_{\tau=\max \{0, t-w+1\}}^{t} x_{\tau \mid t}$. Taking expectations on both sides of the above inequality yields $\mathbb{E}\left[x^{S}(t)\right]=\frac{\sum_{\tau=\max \{0, t-w+1\}}^{t} \mathbb{E}\left[x_{\tau \mid t}\right]}{\max \{w, t+1\}}$. Since $E\left[x_{t \mid \tau}\right]$ are the same for all $t$ and $\tau$ and each solution satisfies the resource constraint of P2, we have

$$
\sum_{t=0}^{T-1} \mathbb{E}\left[x^{S}(t)\right] \leq \Delta .
$$

From Equations (7) and (8), we have Theorem 4.1.

\section{A.4 Proof of Proposition 1}

Assume the relative frequency of $z_{i}$ over the total $T$ time slots is $f_{i} \cdot \mathbf{p}=\left(p_{1}, p_{2}, \ldots, p_{I}\right)$ denotes the system state probability distribution. Similar to Lemma 1 , we have that for $\psi \in(0,1)$, the probability $\operatorname{Pr}\left(\bigcup_{i \in[I]}\left\{\left|p_{i}-f_{i}\right| \leq \psi p_{i}\right\}\right) \geq 1-\sum_{i=1}^{I} 2 e^{-\frac{T \psi^{2} p_{i}}{3}}$. Similar to the proof of Lemma 2, we have that $\eta_{\mathrm{z}}^{*}$ is equal to the objective value of

$$
\begin{array}{ll}
\max _{x_{i}} & T \sum_{i} f_{i} \cdot R\left(x_{i}, z_{i}\right) \\
\text { s.t. } & x_{i} \in \mathcal{A}_{z_{i}} \text { for } i \in\{1,2, \ldots, I\} \\
& T \sum_{i} f_{i} x_{i} \leq \Delta .
\end{array}
$$


When $\left|f_{i}-p_{i}\right| \leq \psi p_{i}, i \in[I]$, similar to the proof for Lemma 2, we have that $\eta_{o p t, \mathrm{p}}$, the optimal objective value of P6 is greater than $\frac{1-\psi}{1+\psi}$ times the optimal objective of Equation (9). That is, $\eta_{o p t, \mathbf{p}} \geq \frac{1-\psi}{1+\psi} \eta^{*}$ when $\left|f_{i}-p_{i}\right| \leq \psi p_{i}, i \in[I]$. Using the same techniques in A.2 and A.3, we can get that $\mathbb{E}\left[\eta^{S}\right] \geq \eta^{*} \cdot \frac{1-\epsilon}{1+\epsilon} \cdot\left(1-\sum_{i=1}^{I} 2 e^{-\frac{w \epsilon^{2} p_{i}}{3}}\right)$ for all $\epsilon \in(0,1)$. Combining the above statement and $\eta_{o p t, \mathrm{p}} \geq \frac{1-\psi}{1+\psi} \eta_{o p t, \mathrm{p}}$ when $\left|f_{i}-p_{i}\right| \leq \psi p_{i}, i \in[I]$, we have that $\mathbb{E}\left[\eta^{S}\right] \geq \eta^{*} \cdot \frac{1-\epsilon}{1+\epsilon} \cdot \frac{1-\psi}{1+\psi} \cdot\left(1-\sum_{i=1}^{I} 2 e^{-\frac{w \epsilon^{2} p_{i}}{3}}\right)$ for all $\epsilon \in(0,1)$ when $\left|f_{i}-p_{i}\right| \leq \psi p_{i}, i \in[I]$.

Since the above statement holds and $\operatorname{Pr}\left(\bigcup_{i \in[I]}\left\{\left|p_{i}-f_{i}\right| \leq \psi p_{i}\right\}\right) \geq 1-\sum_{i=1}^{I} 2 e^{-\frac{T \psi^{2} p_{i}}{3}}$, we have that for $\epsilon, \psi \in(0,1), \operatorname{Pr}\left(\mathbb{E}\left[\eta^{S}\right] \geq \eta^{*} \cdot \frac{1-\epsilon}{1+\epsilon} \cdot \frac{1-\psi}{1+\psi} \cdot\left(1-\sum_{i=1}^{I} 2 e^{-\frac{w \epsilon^{2} p_{i}}{3}}\right)\right) \geq 1-\sum_{i=1}^{I} e^{-\frac{T \psi^{2} p_{i}}{3}}$.

\section{A.5 Proof of Proposition 2}

First, since $\sum_{\tau=t}^{t+\tau-1} x_{t \mid \tau} \leq \frac{\Delta}{T / w}$ holds for $t \in\{1,2, \ldots, T\}$, we have that $\sum_{t=1}^{T} \sum_{\tau=t}^{t+w-1} x_{t \mid \tau} \leq w \cdot \Delta$. That is $\sum_{t=1}^{T} \sum_{\tau=t}^{t+w-1} \frac{1}{w} x_{t \mid \tau}=\Delta$. Since $x_{t}^{S}=\frac{1}{\min \{t, w\}} \sum_{\tau=\max \{1, t-w+1\}}^{t} x_{\tau \mid t}$, we have that

$$
\sum_{t=1}^{T} x_{t}^{S}-\sum_{t=1}^{T} \sum_{\tau=t}^{t+w-1} \frac{1}{w} x_{t \mid \tau} \leq \sum_{t=1}^{w-1} \sum_{\tau=1}^{t} \frac{1}{t} x_{\tau \mid t} \stackrel{(a)}{=} \sum_{t=1}^{w-1} \sum_{\tau=t}^{w-1} \frac{1}{\tau} x_{t \mid \tau} \leq \sum_{t=1}^{w-1} \frac{1}{t} \sum_{\tau=t}^{w-1} x_{t \mid \tau} \leq \sum_{t=1}^{w-1} \frac{1}{t} \frac{\Delta}{T / w} .
$$

(a) holds because $\sum_{j=1}^{n} \sum_{k=j}^{n} a_{j, k}=\sum_{1 \leq j \leq k \leq n} a_{j, k}=\sum_{k=1}^{n} \sum_{j=1}^{k} a_{j, k}$. Since $\sum_{t=1}^{w-1} \frac{1}{t} \leq 1+$ $\int_{t=1}^{w-1} \frac{1}{t}=1+\log (w-1)$ and the above inequality holds, $\sum_{t=1}^{T} x_{t}^{S}-\sum_{t=1}^{T} \sum_{\tau=t}^{t+w-1} \frac{1}{w} x_{t \mid \tau} \leq$ $\sum_{t=1}^{w-1} \frac{1}{t} \frac{\Delta}{T / w} \leq(1+\log (w-1)) \frac{\Delta}{T / w}$. Because $\sum_{t=1}^{T} \sum_{\tau=t}^{t+w-1} \frac{1}{w} x_{t \mid \tau}=\Delta$, we have that $\sum_{t=1}^{T} x^{S}(t) \leq$ $(1+\log (w-1)) \frac{\Delta}{T / w}+\Delta$.

\section{B PERFORMANCE ANALYSIS FOR CPA}

\section{B.1 Lemma 3}

First, for any system state sequence $\mathrm{z} \in \mathrm{Z}$, we generate a sequence of revenue functions $\left\{g^{0}, g^{2}, \ldots, g^{N-1}\right\}$. Second, we construct an online problem P8 and propose an algorithm, called $C P A 1$, for P8. Relying on P8 and CPA1, we find the maximum amount of inventory that may be used by $C P A(\pi)$, which is a function of $\pi$. Finally, we fix the maximum resource to be $\Delta$ and solve the feasible region of $\pi$.

For a system state sequence $\mathrm{z} \in \mathrm{Z}$, we construct a sequence of revenue functions using the following method. For $n \in\{0,1, \ldots, N-1\}$, we define a function $g^{n}: \mathcal{A}_{n}=\left[0, \sum_{t=n w+1}^{n w+w} \delta_{z_{t}} / w\right] \rightarrow$ $\mathbb{R}$. For $x \in \mathcal{A}_{n}, g^{n}(x)$ equals the optimal objective value of $\mathrm{P} 7$ :

$$
\begin{aligned}
\max _{x_{\tau}, \tau \in \mathcal{T}_{n}} & \frac{1}{w} \sum_{\tau=n w+1}^{n w+w} R\left(x_{\tau}, z_{\tau}\right) \\
\text { s.t. } & x_{\tau} \in \mathcal{A}_{z_{\tau}} \text { for } \tau \in \mathcal{T}_{n} \\
& \frac{1}{w} \sum_{\tau=n w+1}^{n w+w} x_{\tau} \leq x .
\end{aligned}
$$

Function $g^{n}(\cdot)$ has some properties that we desire, as shown in Lemma 3.

Lemma 3. $g^{n}(x)$ for $n \in\{0,1, \ldots, N-1\}$ satisfies the following properties:

- $g^{n}(x)$ is concave, continuous, increasing, piecewise differentiable in $\mathcal{A}_{n}$.

- $g^{n}(0)=0$.

- $p_{g^{n}} \triangleq \lim _{x \rightarrow 0^{+}} \frac{g^{n}(x)}{x} \in[m, M]$. 
We prove Lemma 3 by exploiting the dual problem of P7. We omit the proof of Lemma 3 due to space limitations.

\section{B.2 Lemma 5}

For $t \in \mathcal{T}_{n}$, let $g_{t}\left(x_{t}\right) \triangleq g^{n}\left(x_{t}\right)$. If $t \in \mathcal{T}_{n}$, we define $N^{t} \triangleq n$. We use $p_{g}(t)$ to denote the base price of $g_{t}\left(x_{t}\right)$, which is equal to $\left.\frac{\partial g_{t}\left(x_{t}\right)}{\partial x_{t}}\right|_{x_{t}=0}=p^{N^{t}}$.

After having $g_{t}(x)$ and its properties, we consider the following online optimization problem P8:

$$
\begin{array}{ll}
\max _{x_{t}} & \sum_{t=1}^{T} g_{t}\left(x_{t}\right) \\
\text { s.t. } & x_{t} \in \mathcal{A}_{N^{t}} \text { for } t \in\{1,2, \ldots, T\} \\
& \sum_{t=1}^{T} x_{t} \leq \Delta .
\end{array}
$$

$\mathrm{P} 8$ is an online problem similar to $\mathrm{P} 1$. The revenue function at time slot $t$ of $\mathrm{P} 8$ is $g_{t}\left(x_{t}\right)$, which is generated by $R\left(x_{\tau}, z_{\tau}\right), \tau \in \mathcal{T}_{N^{t}}$. Since $g_{t}(\cdot)$ is identical for $t \in \mathcal{T}_{n}$, the system controller of P8 can get access to $g_{t}(\cdot), t \in \mathcal{T}_{n}$ at the beginning of $t=n w+1$.

For P8, we apply an algorithm, called CPA1, which is similar to $C P A$. We use $C P A 1(\pi)$ to denote $C P A 1$ with parameter $\pi$, where $\pi>1$. CPA $1(\pi)$ makes control actions as follows. At the beginning of each time slot $n w+1$, which is the beginning of $\mathcal{T}_{n}$, the system controller of P8 solves the convex optimization problem P9:

$$
\begin{aligned}
\min _{x_{t}, t \in \mathcal{T}_{n}} & \sum_{t=n w+1}^{n w+w} x_{t} \\
\text { s.t. } & \sum_{t=n w+1}^{n w+w} g_{t}\left(x_{t}\right) \geq \frac{1}{\pi}\left(\hat{\eta}^{*}((n+1) w)-\hat{\eta}^{*}(n w)\right) \\
& x_{t} \in \mathcal{A}_{n} \text { for } t \in \mathcal{T}_{n},
\end{aligned}
$$

where $n \in\{0,1, \ldots, N-1\}, \hat{\eta}^{*}(0)=0$ and $\hat{\eta}^{*}(t), t \geq 1$ is the optimal objective value of the following convex optimization problem:

$$
\begin{array}{ll}
\max _{x_{\tau}} & \sum_{\tau=1}^{t} g_{\tau}\left(x_{\tau}\right) \\
\text { s.t. } & x_{\tau} \in \mathcal{A}_{n^{\tau}}, \tau \in\{1, \ldots, t\} \\
& \sum_{\tau=1}^{t} x_{\tau} \leq \Delta .
\end{array}
$$

We denote the optimal solution of P9 by $\bar{x}_{t}^{\pi}$, where $t \in \mathcal{T}_{n}$ and $n \in\{0,1, \ldots, N-1\}$. Then, at time slot $t$, under $C P A 1(\pi)$, the system controller of P8 chooses $\bar{x}_{t}^{\pi}$ as the control action of P8. Also, note that the constraint is tight in P9 for any optimal solution, i.e., $\sum_{\tau=n w+1}^{n w+w} R\left(\bar{x}_{\tau}^{\pi}, z_{\tau}\right)=$ $\frac{1}{\pi}\left(\eta^{*}((n+1) w)-\eta^{*}(n w)\right)$ holds, because we can have a solution better than $\bar{x}_{t}^{\pi}, t \in \mathcal{T}_{n}$ if we have $\sum_{\tau=n w+1}^{n w+w} g_{\tau}\left(\bar{x}_{\tau}^{\pi}\right)>\frac{1}{\pi}\left(\hat{\eta}^{*}((n+1) w)-\hat{\eta}^{*}(n w)\right)$.

Lemma 4. $\eta^{*}(n w)=\hat{\eta}^{*}(n w)$ for $n \in\{0, \ldots, N\}$.

We omit the proof of Lemma 4 . We can prove Lemma 4 by showing $\eta^{*}(n w) \geq \hat{\eta}^{*}(n w)$ and $\eta^{*}(n w) \leq \hat{\eta}^{*}(n w)$, respectively. 
We say that $C P A 1(\pi)$ is feasible for P8, if $\max _{\mathbf{z} \in \mathbf{Z}} \sum_{t=1}^{T} \bar{x}_{t}^{\pi} \leq \Delta$.

Based on the definition P8 and CPA1 for it, we have Lemma 5, which is a connection between $\mathrm{P} 1$ and $\mathrm{P} 8$.

LEMma 5. If $C P A 1(\pi)$ is feasible for $P 8, C P A(\pi)$ will be feasible for $P 1$.

Proof. Let $\Delta_{n} \triangleq \sum_{\tau \in \mathcal{T}_{n}} x_{\tau}^{\pi}$, which is the amount of resource of P1 consumed over $\tau \in \mathcal{T}_{n}$ under $C P A(\pi)$. Let $\Delta_{n}^{\prime} \triangleq \sum_{\tau \in \mathcal{T}_{n}} \bar{x}_{\tau}^{\pi}$, which is the amount of resource of P8 consumed over $\tau \in \mathcal{T}_{n}$ under $C P A 1(\pi)$. Since $g_{\tau}(\cdot)$ is identical for $\tau \in \mathcal{T}_{n}$, WLOG, we assume $\bar{x}_{\tau}^{\pi}$ are identical for $\tau \in \mathcal{T}_{n}$ because if $\bar{x}_{\tau}^{\pi}$ are not equal for $\tau \in \mathcal{T}_{n}$, we can generate another optimal solution $\ddot{x}_{\tau}^{\pi}$ for P9 by letting $\ddot{x}_{\tau}^{\pi}=\sum_{\tau^{\prime} \in \mathcal{T}_{n}} \bar{x}^{\pi}\left(\tau^{\prime}\right) / w, \tau \in \mathcal{T}_{n}$. Thus, the revenue of P8 under $C P A 1(\pi)$ over $\tau \in \mathcal{T}_{n}$ is $w \cdot g_{\tau}\left(\Delta_{n}^{\prime} / w\right)$. According to the definition of $g_{t}(x)$, under $C P A 1(\pi)$, the revenue of P8 over $t \in \mathcal{T}_{n}$ is the optimal objective value of the following convex optimization problem:

$$
\begin{array}{ll}
\max _{x_{t}} & \frac{1}{\pi} \sum_{t=n w+1}^{n w+w} R\left(x_{t}, z_{t}\right) \\
\text { s.t. } & x_{t} \in \mathcal{A}_{z_{t}} \text { for } t \in \mathcal{T}_{n} \\
& \sum_{t=n w+1}^{n w+w} x_{t} \leq \Delta_{n}^{\prime} .
\end{array}
$$

If $\Delta_{n}^{\prime}<\Delta_{n}$, then there exists a solution $x_{t}, t \in \mathcal{T}_{n}$, where $\sum_{t \in \mathcal{T}_{n}} x_{t}=\Delta_{n}^{\prime}<\Delta_{n}=\sum_{t \in \mathcal{T}_{n}} x_{t}^{\pi}$ and $\sum_{t \in \mathcal{T}_{n}} R\left(x_{t}, z_{t}\right)=\frac{1}{\pi}\left(\hat{\eta}^{*}(n w+w)-\hat{\eta}^{*}(n w)\right)=\frac{1}{\pi}\left(\eta^{*}(n w+w)-\eta^{*}(n w)\right)$, which contradicts the fact that $x_{t}^{\pi}$ is the optimal solution of P4. The last equality comes from Lemma 4 . Therefore, $\Delta_{n}^{\prime} \geq \Delta_{n}$.

Since $\Delta_{n}^{\prime} \geq \Delta_{n}$, we have $\sum_{t \in[T]} x_{t}^{\pi}=\sum_{n=0}^{N-1} \Delta_{n} \leq \sum_{n=0}^{N-1} \Delta_{n}^{\prime}=\sum_{t \in[T]} \bar{x}_{t}^{\pi} \leq \Delta$. Therefore, if $C P A 1(\pi)$ is feasible for P8, CPA $(\pi)$ will be feasible for P1.

\section{B.3 Lemma 6}

Since if $C P A 1(\pi)$ is feasible for P8 $C P A(\pi)$ is feasible for P1, we now focus on P8 and CPA1 only. Next, we get a feasible region of $C P A 1(\pi)$ relying on $C R$-Pursuit $(\pi)$ [23] applied to P8. CR-Pursuit $(\pi)$ chooses the control action at time slot $t$ as $\check{x}_{t}^{\pi}$, which is the optimal solution of the following convex optimization problem:

$$
\begin{array}{ll}
\min _{x_{t}} & x_{t} \\
\text { s.t. } & g_{t}\left(x_{t}\right) \geq \frac{1}{\pi}\left(\hat{\eta}^{*}(t+1)-\hat{\eta}^{*}(t)\right) \\
& x(t) \in\left[0, \sum_{\tau \in \mathcal{T}_{N^{t}}} \delta_{z_{\tau}} / w\right] .
\end{array}
$$

Note that the first constraint is tight for any optimal solution, i.e., $g_{t}\left(\check{x}_{t}^{\pi}\right)=\frac{1}{\pi}\left(\hat{\eta}^{*}(t+1)-\hat{\eta}^{*}(t)\right)$ holds, because otherwise there can be a solution better than $\check{x}_{t}^{\pi}$ by decreasing any non-zero $\check{x}_{t}^{\pi}$.

LEMMA 6. If CR-Pursuit( $\pi)$ is feasible for P8, CPA1 $(\pi)$ is feasible for P8.

Proof. We apply the CR-Pursuit $(\pi)$ to the online problem P8. It is clear that the revenue over $t \in \mathcal{T}_{n}$ under the CR-Pursuit $(\pi)$ is $\sum_{t \in \mathcal{T}_{n}} g_{t}\left(\check{x}_{t}^{\pi}\right)=\frac{1}{\pi}\left(\hat{\eta}^{*}(n w+w)-\hat{\eta}^{*}(n w)\right)$, which is equal to the revenue over $t \in \mathcal{T}_{n}$ under the $C P A 1(\pi)$. Since $\bar{x}_{t}^{\pi}, t \in \mathcal{T}_{n}$ is the optimal solution of P9, we have $\sum_{t \in \mathcal{T}_{n}} \bar{x}^{\pi}(t) \leq \sum_{t \in \mathcal{T}_{n}} \check{x}_{t}^{\pi}$. Summing it over $n \in\{0,1, \ldots, N-1\}$, we have that $\sum_{t=1}^{T} \bar{x}_{t}^{\pi} \leq$ $\sum_{t=1}^{T} \check{x}_{t}^{\pi}$. If CR-Pursuit is feasible, we have $\sum_{t=1}^{T} \check{x}_{t}^{\pi} \leq \Delta$. Since $\sum_{t=1}^{T} \bar{x}_{t}^{\pi} \leq \sum_{t=1}^{T} \check{x}_{t}^{\pi}$, if CR-Pursuit is feasible, we have $\sum_{t=1}^{T} \bar{x}_{t}^{\pi} \leq \sum_{t=1}^{T} \check{x}_{t}^{\pi} \leq \Delta$. That is, CPA1 is feasible for P8 if CR-Pursuit is feasible for P8. 


\section{B.4 Lemma 7 and Lemma 8}

Next, we focus on P8 and CR-Pursuit $(\pi)$ for it. Because Lemma 3 in Appendix B.1 holds, P8 is similar to the OOIC problem in [23] and we use some results from [23] shown in Lemma 7 and Lemma 8.

Lemma 7. For P8 under CR-Pursuit( $\pi)$, we have $\check{x}_{t}^{\pi} \leq c \frac{g_{t}\left(\check{x}_{t}^{\pi}\right)}{p_{g}(t)}, \forall t \in\{1,2, \ldots, T\}$, where $c=$ $\max _{\mathrm{z} \in \mathrm{Z}} \frac{p(t)}{R\left(\delta_{z_{t}}, z_{t}\right) / \delta_{z_{t}}}$.

Note that base price $p_{g}(t)=\lim _{x_{t} \rightarrow 0^{+}} \frac{g_{t}\left(x_{t}\right)}{x_{t}} \in[m, M]$, and $p(t)=\lim _{x_{t} \rightarrow 0^{+}} \frac{R\left(x_{t}, z_{t}\right)}{x_{t}} \in[m, M]$. As stated in [23], the $c$ is bounded and small for many interesting problems. By exploiting Lemma 3 , we can prove Lemma 7. The proof of Lemma 7 is similar to the proof of Lemma 9 in [23], so we omit it.

Lemma 8. For P8 under CR-Pursuit $(\pi)$ and any threshold price $p \in[m, M]$, we have

$$
\sum_{\left\{t: p_{g}(t) \leq p\right\}} g_{t}\left(\check{x}_{t}^{\pi}\right) \leq \frac{1}{\pi} p \Delta .
$$

The proof of Lemma 8 is similar to the proof of Lemma 10 in [23], so we omit it.

\section{B.5 Lemma 9}

Lemma 7 in Appendix B.4 shows that, for P8, under CR-Pursuit $(\pi)$, the amount of resource consumed in time slot $t$ is less than a term related to the revenue at time slot $t$ and the base price $p_{g}(t)$. Lemma 8 in Appendix A12 shows that, for a given threshold $p$, the total revenue of all the slots with $p_{g}(t)<p$ is less than the $\frac{1}{\pi} p \times \Delta$. Combining Lemma 7 and Lemma 8 , we are able to get an upper bound of the amount of resource of P8 under CR-Pursuit as shown in Lemma 9. Lemma 9 is a crucial step to the final result.

LEMMA 9. For P8, under CR-Pursuit( $\pi)$, we have

$$
\sum_{t=1}^{T} \frac{g_{t}\left(\check{x}_{t}^{\pi}\right)}{p_{g}(t)} \leq \frac{\Delta}{\pi} \times\left(1+\left(\frac{T}{w}-1\right)\left(1-\theta^{-\frac{1}{\frac{T}{w}-1}}\right)\right) .
$$

Proof. According to the definitions of $g_{t}(\cdot)$ and $p^{n}$, and Lemma 3, we have that $p_{g}(t)=p^{n}$ for $t \in \mathcal{T}_{n}$ and $n \in\{0,1, \ldots, N-1\}$. Assume $p^{n}, n \in\{0,1, \ldots, N-1\}$ take $k$ different values, i.e., $q_{1}, q_{2}, \ldots, q_{k}$. WLOG, we assume $q_{1}<q_{2}<\ldots<q_{k}$. Define $y_{i}=\sum_{\left\{t: p_{g}(t)=q_{i}\right\}} \frac{\pi}{\Delta} g_{t}\left(\check{x}_{t}^{\pi}\right)$. We have

$$
\sum_{t=1}^{T} \frac{g_{t}\left(\check{x}_{t}^{\pi}\right)}{p_{g}(t)}=\frac{\Delta}{\pi} \sum_{i=1}^{k} \frac{y_{i}}{q_{i}} .
$$

Because of Lemma 8 in Appendix B.4, we have $\sum_{j=1}^{i} y_{j} \leq q_{i}$. Then, we consider the following problem:

$$
\begin{array}{ll}
\max _{y_{i}} & \sum_{i=1}^{k} \frac{y_{i}}{q_{i}} \\
\text { s.t. } & \sum_{j=1}^{i} y_{j} \leq q_{i}, \text { for } i \in\{1,2, \ldots, k\} \\
& y_{i} \geq 0, \text { for } i \in\{1,2, \ldots, k\} .
\end{array}
$$


Use $\mu_{i}>0, i \in[k]$ to denote the dual variables of P11. Similar to Equations (23) and (24) in [23], the KKT conditions of P11 is as follows:

$$
\begin{gathered}
\frac{1}{q_{i}}-\sum_{j=i}^{k} \mu_{j}=0, \forall i \in[k], \\
\mu_{i}\left(q_{i}-\sum_{j=1}^{i} y_{j}\right)=0, \forall i \in[k], \\
u_{i} \geq 0, \forall i \in[k], \\
y_{i} \geq 0 . \forall i \in[k] .
\end{gathered}
$$

From Equation (10), we have $\mu_{i}>0, \forall i \in[k]$ because of the fact that $q_{i}>q_{i-1}$. Because $\mu_{i}>0, \forall i \in$ $[k]$ and Equation (11) holds, we have $\left(q_{i}-\sum_{j=1}^{i} y_{j}\right)=0, \forall i \in[k]$. That is, the optimal solution of $\mathrm{P} 11$ is $y_{i}=q_{i}-q_{i-1}, i \in[k]$ and the optimal objective value of P11 is $\sum_{i=1}^{k} \frac{q_{i}-q_{i-1}}{q_{i}}$, where $q_{0}=0$. That is,

$$
\sum_{t=1}^{T} \frac{g_{t}\left(\check{x}_{t}^{\pi}\right)}{p_{g}(t)}=\frac{\Delta}{\pi} \sum_{i=1}^{k} \frac{y_{i}}{q_{i}} \leq \frac{\Delta}{\pi} \sum_{i=1}^{k} \frac{q_{i}-q_{i-1}}{q_{i}} .
$$

Since $m<q_{1}<q_{2}<\cdots<q_{k}<M$, we then consider the following problem:

$$
\max _{m<q_{1}<q_{2}<\cdots<q_{k}<M} \sum_{i=1}^{k} \frac{q_{i}-q_{i-1}}{q_{i}}=\max _{m<q_{1}<q_{2}<\cdots<q_{k}<M} k-\sum_{i=2}^{k} \frac{q_{i-1}}{q_{i}} .
$$

Applying the AM-GM inequality, we have

$$
\max _{m<q_{1}<q_{2}<\cdots<q_{k}<M} k-\sum_{i=2}^{k} \frac{q_{i-1}}{q_{i}} \leq k-(k-1)\left(\frac{q_{1}}{q_{2}} \cdot \frac{q_{2}}{q_{3}} \cdot \ldots \cdot \frac{q_{k-1}}{q_{k}}\right)^{1 /(k-1)}
$$

with equality if and only if $\frac{q_{1}}{q_{2}}=\frac{q_{2}}{q_{3}}=\cdots=\frac{q_{k-1}}{q_{k}}$. Then,

$$
\begin{aligned}
& \max _{m<q_{1}<q_{2}<\cdots<q_{k}<M} k-\sum_{i=2}^{k} \frac{q_{i-1}}{q_{i}}=k-(k-1)\left(\frac{q_{1}}{q_{2}} \cdot \frac{q_{2}}{q_{3}} \cdot \ldots \cdot \frac{q_{k-1}}{q_{k}}\right)^{1 /(k-1)} \\
& \leq k-(k-1)\left(\frac{m}{M}\right)^{1 /(k-1)}=1+(k-1)\left(1-\theta^{-1 /(k-1)}\right) .
\end{aligned}
$$

$1+(k-1)\left(1-\theta^{-1 /(k-1)}\right)$ increases as $k$ increases because $\frac{d\left(x\left(1-\theta^{-1 / x}\right)\right)}{d x}=\left(1-\theta^{-1 / x}\right)-$ $(1 / x) \theta^{-1 / x} \log (\theta)>0$ when $x>0$ and $\theta>1$. Since $k$ is the number of different values of $p^{n}, n \in\{0,1, \ldots, N-1\}$, we have $k \leq N=\frac{T}{w}$. Therefore, from Equations (12), (13), (14), and (15), we have $\sum_{t=1}^{T} \frac{g_{t}\left(\check{x}_{t}^{\pi}\right)}{p_{g}(t)} \leq \frac{\Delta}{\pi}\left(1+(T / w-1)\left(1-\theta^{-1 /(T / w-1)}\right)\right)$.

In what follows, we show the competitive ratio of CR-Pursuit for P8.

Lemma 10. For $\pi \geq c \cdot\left(1+(T / w-1) \cdot\left(1-\theta^{-1 /(T / w-1)}\right)\right)$, CR-Pursuit $(\pi)$ is feasible for P8.

Proof. Lemma 7 in Appendix B.4 says that $\sum_{t=1}^{T} \check{x}_{t}^{\pi} \leq \sum_{t=1}^{T} c \frac{g_{t}\left(\check{x}_{t}^{\pi}\right)}{p_{g}(t)}$. Since Lemma 9 in Appendix B.5 holds, we have that $\sum_{t=1}^{T} \check{x}_{t}^{\pi} \leq \sum_{t=1}^{T} c \frac{g_{t}\left(\check{x}_{t}^{\pi}\right)}{p_{g}(t)} \leq \frac{\Delta}{\pi} \times c \times\left(1+(T / w-1)\left(1-\theta^{-1 /(T / w-1)}\right)\right)$. For $\pi \geq c\left(1+(T / w-1)\left(1-\theta^{-1 /(T / w-1)}\right)\right)$, we have $\sum_{t=1}^{T} \check{x}_{t}^{\pi} \leq \Delta$. 


\section{B.6 Proof of Theorem 4.2}

Combining Lemma 5, Lemma 6, and Lemma 10 as follows, we get the main result of Theorem 4.2. Because CR-Pursuit $(\pi)$ is feasible for P8 when $\pi \geq c\left(1+(T / w-1)\left(1-\theta^{-1 /(T / w-1)}\right)\right)$, we have from Lemma 10 and Lemma 6 that $C P A 1(\pi)$ is feasible for P8 when $\pi \geq c\left(1+(T / w-1)\left(1-\theta^{-1 /(T / w-1)}\right)\right)$. Because Lemma 5 holds and CPA1 $(\pi)$ is feasible for P8 when $\pi \geq c\left(1+(T / w-1)\left(1-\theta^{-1 /(T / w-1)}\right)\right)$, we have that $C P A(\pi)$ is feasible and $\pi$-competitive for $\mathrm{P} 1$ when $\pi \geq c\left(1+(T / w-1)\left(1-\theta^{-1 /(T / w-1)}\right)\right)$.

\section{REFERENCES}

[1] Jacob D. Abernethy, Elad Hazan, and Alexander Rakhlin. 2009. Competing in the dark: An efficient algorithm for bandit linear optimization. Conference on Learning Theory, Retrieved from https://repository.upenn.edu/statistics papers/110.

[2] Maha Achour. 2002. Simulating atmospheric free-space optical propagation: rainfall attenuation. In Free-Space Laser Communication Technologies XIV, Vol. 4635. International Society for Optics and Photonics, 192-201.

[3] Maher C. Al Naboulsi, Herve Sizun, and Frederique de Fornel. 2004. Fog attenuation prediction for optical and infrared waves. Optical Engineering 43, 2 (2004), 319-330.

[4] Sanjeev Arora, Elad Hazan, and Satyen Kale. 2012. The multiplicative weights update method: A meta-algorithm and applications. Theory of Computing 8, 1 (2012), 121-164.

[5] Moshe Babaioff, Nicole Immorlica, David Kempe, and Robert Kleinberg. 2008. Online auctions and generalized secretary problems. ACM SIGecom Exchanges 7, 2 (2008), 7.

[6] O. Bouchet, T. Marquis, M. Chabane, M. Alnaboulsi, and H. Sizun. 2005. FSO and quality of service software prediction. In Free-Space Laser Communications V, Vol. 5892. International Society for Optics and Photonics, 589204.

[7] Niangjun Chen, Joshua Comden, Zhenhua Liu, Anshul Gandhi, and Adam Wierman. 2016. Using predictions in online optimization: Looking forward with an eye on the past. ACM SIGMETRICS Performance Evaluation Review 44, 1 (2016), 193-206.

[8] Joshua Comden, Sijie Yao, Niangjun Chen, Haipeng Xing, and Zhenhua Liu. 2019. Online optimization in cloud resource provisioning: Predictions, regrets, and algorithms. Proceedings of the ACM on Measurement and Analysis of Computing Systems 3, 1 (2019), 16.

[9] Thomas M. Cover. 1999. Elements of Information Theory. John Wiley \& Sons.

[10] Robert Crane. 1980. Prediction of attenuation by rain. IEEE Transactions on Communications 28, 9 (1980), $1717-1733$.

[11] Peter Damaschke, Phuong Hoai Ha, and Philippas Tsigas. 2009. Online search with time-varying price bounds. Algorithmica 55, 4 (2009), 619-642.

[12] Paul DeMaio. 2009. Bike-sharing: History, impacts, models of provision, and future. fournal of Public Transportation 12,4 (2009), 3.

[13] Nachiket Deo and Mohan M. Trivedi. 2018. Convolutional social pooling for vehicle trajectory prediction. In Proceedings of the IEEE Conference on Computer Vision and Pattern Recognition Workshops. 1468-1476.

[14] Mingyang Du, Lin Cheng, Xuefeng Li, and Fang Tang. 2020. Static rebalancing optimization with considering the collection of malfunctioning bikes in free-floating bike sharing system. Transportation Research Part E: Logistics and Transportation Review 141 (2020), 102012.

[15] Ran El-Yaniv, Amos Fiat, Richard M. Karp, and Gordon Turpin. 2001. Optimal search and one-way trading online algorithms. Algorithmica 30, 1 (2001), 101-139.

[16] Moran Feldman and Rico Zenklusen. 2018. The submodular secretary problem goes linear. SIAM fournal on Computing 47, 2 (2018), 330-366.

[17] J. Gao, M. Ito, and N. Shiratori. 2016. Optimal scheduling for incentive WiFi offloading under energy constraint. In 2016 IEEE 27th Annual International Symposium on Personal, Indoor, and Mobile Radio Communications (PIMRC'16). 1-6. https://doi.org/10.1109/PIMRC.2016.7794868

[18] Y. Guo, Q. Yang, F. Fu, and K. S. Kwak. 2015. Quality-oriented rate control and resource allocation in dynamic OFDMA networks. In 2015 IEEE Global Communications Conference (GLOBECOM’15). 1-6. https://doi.org/10.1109/GLOCOM. 2015.7417177

[19] Mohammad H. Hajiesmaili, Chi-Kin Chau, Minghua Chen, and Longbu Huang. 2016. Online microgrid energy generation scheduling revisited: The benefits of randomization and interval prediction. In Proceedings of the 7th International Conference on Future Energy Systems. ACM, 1.

[20] Zhenning Kong and Edmund M. Yeh. 2008. Connectivity and latency in large-scale wireless networks with unreliable links. In IEEE INFOCOM 2008-The 27th Conference on Computer Communications. IEEE, 11-15.

[21] Paul W. Kruse, Laurence D. McGlauchlin, and Richmond B. McQuistan. 1962. Elements of Infrared Technology: Generation, Transmission and Detection. New York: Wiley 
[22] Minghong Lin, Zhenhua Liu, Adam Wierman, and Lachlan L.H. Andrew. 2012. Online algorithms for geographical load balancing. In 2012 International Green Computing Conference (IGCC'12). IEEE, 1-10.

[23] Qiulin Lin, Hanling Yi, John Pang, Minghua Chen, Adam Wierman, Michael Honig, and Yuanzhang Xiao. 2019. Competitive online optimization under inventory constraints. Proceedings of the ACM on Measurement and Analysis of Computing Systems 3, 1 (2019), 10.

[24] Junming Liu, Leilei Sun, Weiwei Chen, and Hui Xiong. 2016. Rebalancing bike sharing systems: A multi-source data smart optimization. In Proceedings of the 22nd ACM SIGKDD International Conference on Knowledge Discovery and Data Mining. 1005-1014.

[25] Y. Liu, Y. Cui, and X. Wang. 2015. Connectivity and transmission delay in large-scale cognitive radio ad hoc networks with unreliable secondary links. IEEE Transactions on Wireless Communications 14, 12 (Dec. 2015), 7016-7029. https: //doi.org/10.1109/TWC.2015.2463816

[26] Hengyu Long, Yongpan Liu, Yiqun Wang, Robert P. Dick, and Huazhong Yang. 2009. Battery allocation for wireless sensor network lifetime maximization under cost constraints. In 2009 IEEE/ACM International Conference on ComputerAided Design-Digest of Technical Papers. IEEE, 705-712.

[27] Mohammad Mahdian, Hamid Nazerzadeh, and Amin Saberi. 2007. Allocating online advertisement space with unreliable estimates. In Proceedings of the 8th ACM Conference on Electronic Commerce. ACM, 288-294.

[28] Farukh Nadeem, Vaclav Kvicera, Muhammad Saleem Awan, Erich Leitgeb, Sajid Sheikh Muhammad, and Gorazd Kandus. 2009. Weather effects on hybrid FSO/RF communication link. IEEE fournal on Selected Areas in Communications 27, 9 (2009), 1687-1697.

[29] Michael J. Neely. 2010. Stochastic network optimization with application to communication and queueing systems. Synthesis Lectures on Communication Networks 3, 1 (2010), 1-211.

[30] M. Prist, A. Freddi, S. Longhi, A. Monteriù, and P. Antonini. 2016. Wireless sensor network based management system for electric bicycle-sharing. In 2016 IEEE 16th International Conference on Environment and Electrical Engineering (EEEIC'16). 1-6.

[31] Aviad Rubinstein. 2016. Beyond matroids: Secretary problem and prophet inequality with general constraints. In Proceedings of the 48th Annual ACM Symposium on Theory of Computing. ACM, 324-332.

[32] X. Shang, Y. Liu, Y. Mao, Z. Liu, and Y. Yang. 2020. Greening reliability of virtual network functions via online optimization. In 2020 IEEE/ACM 28th International Symposium on Quality of Service (IWQoS'20). 1-10.

[33] Rahul Singh, Upendra Sharma, Emmanuel Cecchet, and Prashant Shenoy. 2010. Autonomic mix-aware provisioning for non-stationary data center workloads. In Proceedings of the 7th International Conference on Autonomic Computing. ACM, 21-30.

[34] S. Xu, G. Zhu, C. Shen, S. Li, and Z. Zhong. 2016. Delay-aware dynamic resource management for high-speed railway wireless communications. In 2016 IEEE 83rd Vehicular Technology Conference (VTC Spring'16). 1-5. https://doi.org/10. 1109/VTCSpring.2016.7504187

[35] H. Yi, Q. Lin, and M. Chen. 2019. Balancing cost and dissatisfaction in online EV charging under real-time pricing. In IEEE INFOCOM 2019 - IEEE Conference on Computer Communications. 1801-1809.

[36] Wenming Zhang, Yinfeng Xu, Feifeng Zheng, and Yucheng Dong. 2012. Optimal algorithms for online time series search and one-way trading with interrelated prices. Journal of Combinatorial Optimization 23, 2 (2012), 159-166.

[37] Zhen-wei Zhao, Ming-gao Zhang, and Zhen-sen Wu. 2001. Analytic specific attenuation model for rain for use in prediction methods. International fournal of Infrared and Millimeter Waves 22, 1 (2001), 113-120.

[38] Pengzhan Zhou, Cong Wang, Yuanyuan Yang, and Xin Wei. 2020. E-Sharing: Data-driven online optimization of parking location placement for dockless electric bike sharing. In 2020 IEEE 40th International Conference on Distributed Computing Systems (ICDCS'20). IEEE, 474-484.

[39] Xuan Kelvin Zou, Jeffrey Erman, Vijay Gopalakrishnan, Emir Halepovic, Rittwik Jana, Xin Jin, Jennifer Rexford, and Rakesh K. Sinha. 2015. Can accurate predictions improve video streaming in cellular networks? In Proceedings of the 16th International Workshop on Mobile Computing Systems and Applications. ACM, 57-62.

Received November 2020; revised April 2021; accepted June 2021 